\title{
CaHDZ27, a Homeodomain-Leucine Zipper I Protein, Positively Regulates the Resistance to Ralstonia solanacearum Infection in Pepper
}

\author{
Shaoliang Mou, ${ }^{1,2}$ Zhiqin Liu, ${ }^{1,3}$ Feng Gao, ${ }^{1,2}$ Sheng Yang, ${ }^{1,3}$ Meixia Su, ${ }^{2}$ Lei Shen,,${ }^{1,3}$ Yang $W u,{ }^{4, \dagger}$ and \\ Shuilin $\mathrm{He}^{1,3, \dagger}$

\begin{abstract}
${ }^{1}$ National Education Ministry Key Laboratory of Plant Genetic Improvement and Comprehensive Utilization, Fujian Agriculture and Forestry University, Fuzhou, Fujian 350002, PR China; ${ }^{2}$ College of Life Science, Fujian Agriculture and Forestry University; ${ }^{3}$ College of Crop Science, Fujian Agriculture and Forestry University; and ${ }^{4}$ College of Life Science, Jinggang Shan University, Ji'an, Jiangxi 343000, PR China
\end{abstract}

Accepted 23 August 2017.

\begin{abstract}
Homeodomain-leucine zipper class I (HD-Zip I) transcription factors have been functionally characterized in plant responses to abiotic stresses, but their roles in plant immunity are poorly understood. Here, a HD-Zip I gene, $\mathrm{CaHZ27}$, was isolated from pepper (Capsicum annum) and characterized for its role in pepper immunity. Quantitative real-time polymerase chain reaction showed that $\mathrm{CaHDZ27}$ was transcriptionally induced by Ralstonia solanacearum inoculation and exogenous application of methyl jasmonate, salicylic acid, or ethephon. The CaHDZ27-green fluorescent protein fused protein was targeted exclusively to the nucleus. Chromatin immunoprecipitation demonstrated that $\mathrm{CaHDZ27}$ bound to the 9-bp pseudopalindromic element (CAATAATTG) and triggered $\beta$-glucuronidase expression in a CAATAATTG-dependent manner. Virus-induced gene silencing of $\mathrm{CaHDZ27}$ significantly attenuated the resistance of pepper plants against $R$. solanacearum and downregulated defense-related marker genes, including CaHIR1, CaACO1, CaPR1, CaPR4, CaPO2, and CaBPR1. By contrast, transient overexpression of $\mathrm{CaHDZ27}$ triggered strong cell death mediated by the hypersensitive response and upregulated the tested immunity-associated marker genes. Ectopic $\mathrm{CaHDZ27}$ expression in tobacco enhances its resistance against $R$. solanacearum. These results collectively suggest that $\mathrm{CaHDZ27}$ functions as a positive regulator in pepper resistance against $R$. solanacearum. Bimolecular fluorescence complementation and coimmunoprecipitation assays indicate that CaHDZ27 monomers bind with each other, and this binding is enhanced significantly by $R$. solanacearum inoculation. We speculate that homodimerization of CaHZ27 might play a role in pepper response to $R$. solanacearum, further direct evidence is required to confirm it.
\end{abstract}

Plants encounter a vast array of potentially phytopathogenic pathogens in their natural environments and have developed sophisticated defense strategies during coevolution with these pathogens. These strategies generally include constitutive chemical and physical barriers along with inducible innate

${ }^{\dagger}$ Corresponding authors: Shuilin He; E-mail: shlhe201304@aliyun.com and Yang Wu; E-mail: 40770249@qq.com

*The $\boldsymbol{e}$-Xtra logo stands for "electronic extra" and indicates that six supplementary figures and four supplementary tables are published online.

(c) 2017 The American Phytopathological Society immunity, which includes pathogen-associated molecular patterntriggered immunity and effector-triggered immunity (ETI) (Hein et al. 2009; Jones and Dangl 2006). Inducible innate immunity is largely regulated at the transcriptional level by the actions of many transcriptional factors (TFs), which are generally encoded by large gene families. TFs are important players in regulated gene expression networks. Some studies have suggested that TFs are promising biotechnological targets for engineering-improved plant disease resistance (Goossens et al. 2017; Wang et al. 2015; Xu et al. 2011). To fully exploit these immunity-related TFs, a deeper understanding of their functional roles and mechanisms is required.

HD-Zip proteins contain a DNA-binding homeodomain (HD) and the adjacent leucine zipper (Zip) (Ariel et al. 2007). HD-Zip proteins constitute a large family of plant-specific TFs, with the identification of 55 HD-Zip TFs in corn (Mao et al. 2016), 27 in citrus (Ge et al. 2015), 51 in tomato (Zhang et al. 2014b), 33 in peach (Zhang et al. 2014a), 88 in soybean (Chen et al. 2014), 63 in Populus spp. (Hu et al. 2012), and 47 in Arabidopsis thaliana (Henriksson et al. 2005). HD-Zip protein family members are classified into four subfamilies (HD-Zip I to HD-Zip IV) based on their sequence similarities (Ariel et al. 2007; Henriksson et al. 2005; $\mathrm{Hu}$ et al. 2016; Schena and Davis 1994). The majority of $H D$ Zip genes in different classes are responsible for modulating developmental processes, such as those of vascular tissue, trichomes, cuticle (Müller et al. 2016; Yan et al. 2017), embryogenesis (Roodbarkelari and Groot 2017), leaf polarity (Merelo et al. 2016), and flowering (Kovalchuk et al. 2016). HD-Zip genes also are involved in plant responses to drought (Chen et al. 2014; Harris et al. 2016; Zhao et al. 2014), salinity (Chen et al. 2014; Zhao et al. 2014), and pathogen infection (AbuQamar et al. 2006; Oh et al. 2013).

HD-Zip class I functions by binding to a 9-bp pseudopalindromic DNA sequence (CAATNATTG) in the promoters of their target genes (Johannesson et al. 2001; Palena et al. 1999). HD-Zip I proteins form homodimers and heterodimers with HD-Zip proteins in the same class (Harris et al. 2016; Johannesson et al. 2001; Meijer et al. 2000). Most plant stress-responsive HD-Zip proteins belong to class I. Functional studies of HD-Zip I members indicate that they have important roles in abiotic stress responses to frost (Kovalchuk et al. 2016), flooding (Cabello et al. 2016), and drought (Harris et al. 2016; Romani et al. 2016; Zhao et al. 2014). Several reports suggested that HD-Zip I members are involved in plant responses to pathogen infection. For example, overexpression of the HD-Zip I gene ATHB13 in Arabidopsis leads to broad-spectrum disease resistance (Gao et al. 2014). The tomato HD-Zip I gene H52 is upregulated in response to 
Pseudomonas syringae pv. tomato infection, resulting in an oxidative burst that normally precedes the hypersensitive response (HR) (Mayda et al. 1999). The sunflower HD-Zip I gene $H A H B 4$ is strongly induced by treatment with methyl jasmonate (MeJA) or ethylene (ET). Arabidopsis plants ectopically expressing HAHB4 are sensitive to bacterial infection and reduce levels of SA after infection with Pseudomonas syringae (Manavella et al. 2008). However, the involvement of HD-Zip I proteins in plant immunity is poorly understood.

Pepper (Capsicum annuum) is one of the most important vegetables worldwide. Pepper is a member of the Solanaceae family and is vulnerable to several soilborne diseases, such as bacterial wilt caused by Ralstonia solanacearum, which causes serious yield and economic losses (Flores-Cruz and Allen 2009). The most efficient and sustainable way to overcome pepper pathogenic diseases is to develop cultivars with disease resistance. A deeper understanding of the mechanisms involved in pepper immunity would facilitate the genetic improvement of pepper disease resistance. In the present study, we isolate and functionally characterize the Capsicum annuum HD-Zip I gene CaHDZ27. Our results indicate that CaHDZ27 functions as a positive regulator of pepper's response to $R$. solanacearum infection.

\section{RESULTS}

Sequence analysis of CaHDZ27.

HD-Zip I proteins have been implicated in plant immunity, but no studies have been conducted on HD-Zip I proteins in pepper immunity. To isolate pepper genes associated with immunity, a
cDNA library was constructed using RNA of pepper leaves inoculated with $R$. solanacearum. Random sequencing of this library identified a full-length cDNA containing an open reading frame (ORF) of $891 \mathrm{bp}$ that harbored a homeodomain (HD) and an adjacent leucine zipper (Zip) domain without any other known domains in its deduced amino acid sequence (Fig. 1), suggesting that it was a pepper HD-Zip I protein (Ariel et al. 2007; Brandt et al. 2014). The deduced amino acid sequence of this cDNA was most similar to SIHZ27 (83.39\%), an HDZip I protein family member in tomato (Solanum lycopersicum), we designated it as CaHDZ27. We performed a phylogenetic analysis using the full-length amino acid sequences of CaHDZ27, 22 HD-Zip I proteins from tomato (Zhang et al. 2014b), and 17 from Arabidopsis (Henriksson et al. 2005); the results indicated that CaHDZ27 was most closely related to SIHZ27 and belonged to a subgroup that included only three tomato proteins SIHZ27, SIHZ24, and SlHZ21 (Supplementary Fig. S1). Other subgroups included members from both tomato and Arabidopsis. These results were consistent with a previous report (Zhang et al. 2014b). Alignment analysis of the deduced amino acid sequence of CaHDZ27 and HD-Zip I proteins in other plant species showed the high sequence similarity of CaHDZ27 with other HD-Zip I proteins. Interestingly, we also found a conserved motif in which serine (S) and aspartate (D) are rich (Fig. 1). Therefore, CaHDZ27 is likely an HD-Zip I protein.

\section{CaHDZ27 is transcriptionally upregulated}

by $R$. solanacearum inoculation and exogenous application of salicylic acid (SA), MeJA, or ethephon (ETH).

To test whether $\mathrm{CaHDZ27}$ is involved in pepper immunity, its transcriptional expression was examined by real-time

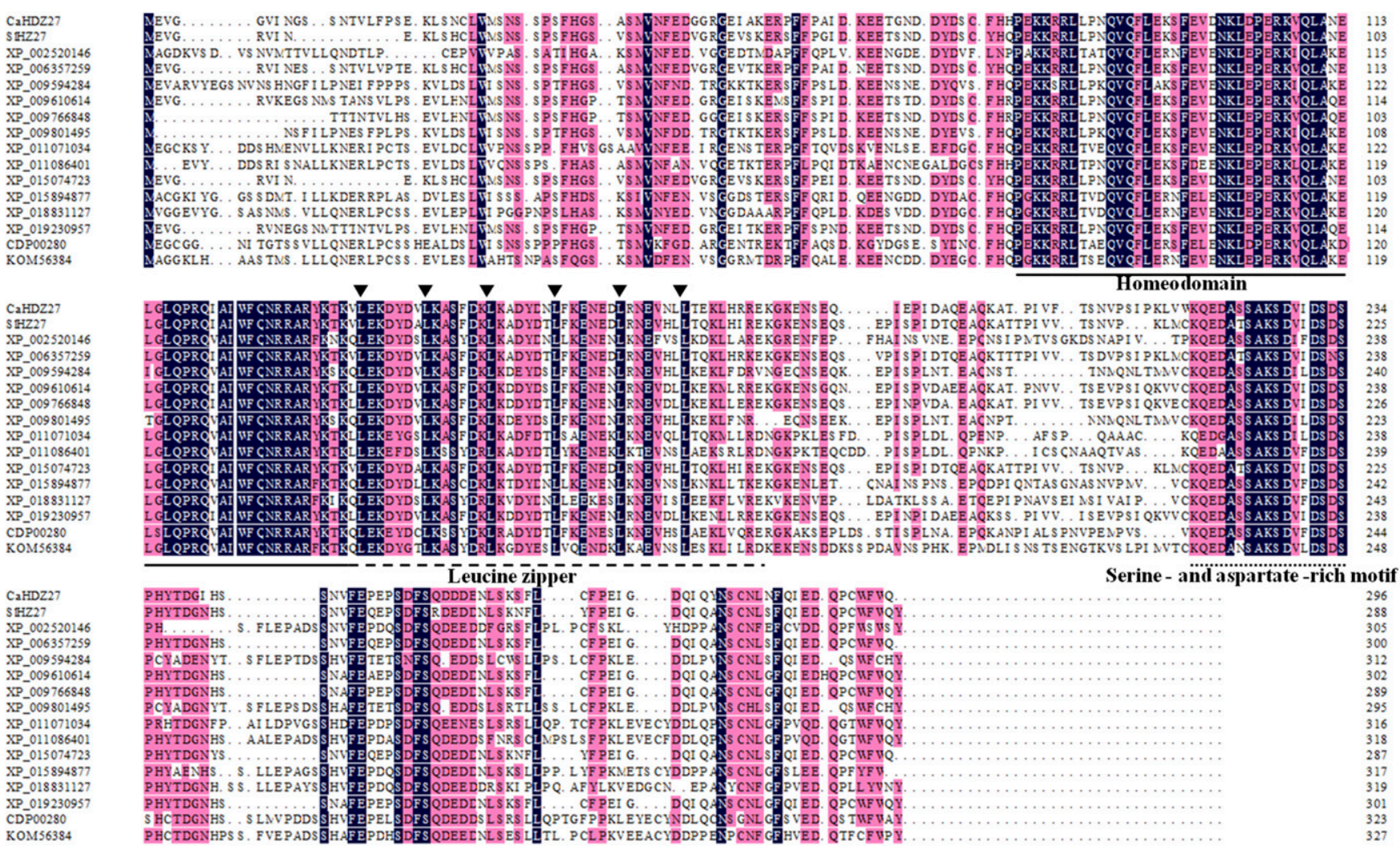

Fig. 1. Alignment of the deduced amino acid sequences of CaHDZ27 and its homolog proteins. Inverted black triangles indicate conserved leucine residues. The homeodomain, adjacent leucine zipper domain and serine (S)- and aspartate (D)-rich motif are indicated by solid, dashed, and dotted lines, respectively. CaHDZ27 homolog proteins were used from potato (Solanum tuberosum, XP_006357259, 88.00\%), the stress-tolerant wild tomato (Solanum pennellii, XP_015074723, 83.67\%), tomato (Solanum lycopersicum, XP_004238764, S1HZ27, 83.67\%), Nicotiana spp. (N. sylvestris, XP_009766848, 81.94\%; N. tomentosiformis, XP_009610614, 80.82\%; N. attenuata, XP_019230957, 82.18\%; N. sylvestris, XP_009801495, 59.42\%, and N. tomentosiformis, XP_009594284, 58.81\%), Coffea canephora (CDP00280, 58.41\%), Ziziphus jujuba (XP_015894877, 57.05\%), Sesamum indicum (XP_011086401, 55.28\% and XP_011071034, 53.42\%), Ricinus communis (XP_002520146, 53.59\%), Juglans regia (XP_018831127, 52.34\%) and Vigna angularis (KOM56384, $50.91 \%)$. 
quantitative polymerase chain reaction (qPCR) analysis of pepper plants inoculated with $R$. solanacearum, the causal agent of bacterial wilt of pepper. The results showed that $\mathrm{CaHDZ27}$ transcript levels were upregulated significantly at 24 and $48 \mathrm{~h}$ postinoculation (Fig. 2A). SA, jasmonic acid (JA), and ET have been implicated as general defense signaling molecules in plant immunity; therefore, we tested the effects of exogenous application of SA, MeJA, or ETH on CaHDZ27 transcript levels in pepper seedlings. Different exogenous hormone concentrations were first investigated to determine suitable concentrations; $1 \mathrm{mM}$ SA, $100 \mu \mathrm{M}$ MeJA, and $100 \mu \mathrm{M}$ are the optimal concentrations for spraying (Supplementary Fig. S2). qPCR analysis showed that MeJA application increased $\mathrm{CaHDZ27}$ transcript levels at 6,12 , and $24 \mathrm{~h}$ posttreatment (hpt), with the peak at $24 \mathrm{hpt}$, compared with those in mock-treated control plants (Fig. 2B). SA application increased $\mathrm{CaHDZ27}$ transcript levels at 1 and $6 \mathrm{hpt}$, compared with those in mock-treated control plants (Fig. 2C). ETH application markedly increased $\mathrm{CaHDZ27}$ transcript levels at 6, 12, and $24 \mathrm{hpt}$, compared with those in mock-treated control plants (Fig. 2D). These combined results indicate that CaHDZ27 transcription is modulated by $R$. solanacearum inoculation and exogenous application of SA, MeJA, and ETH. Therefore, CaHDZ27 may have a functional role in pepper immunity against $R$. solanacearum.

\section{CaHDZ27 protein is localized in the nucleus.}

A nuclear localization sequence (NLS) at amino acid residue 78 to 86 (PEKKRRLLP, cNLS Mapper score 7) was determined using the open source software cNLS Mapper, suggesting that it is targeted to the nucleus. To determine the subcellular localization, $\mathrm{CaHDZ27}$ was fused with the green fluorescent protein $(G F P)$ gene under the control of the $35 \mathrm{~S}$ promoter. This construct (35S::CaHDZ27-GFP) was transformed into Agrobacterium tumefaciens GV3101 and was infiltrated by injection into Nicotiana benthamiana leaves. GFP expression was examined after $48 \mathrm{~h}$, using a Leica confocal microscopy. GFP fluorescence was detected exclusively in the nuclei of cells transiently overexpressing CaHDZ27-GFP, which also were stained with 4',6-diamidino-2-phenylindole (DAPI) (Supplementary Fig. S3). By contrast, GFP fluorescence in control leaves, infiltrated with $35 \mathrm{~S}:: \mathrm{GFP}$, was observed throughout the cells. These results suggest that CaHDZ27 is localized in the nucleus.

\section{CaHDZ27 has DNA-binding and} transactivating activities.

HD-Zip I proteins act as TFs by binding to the pseudopalindromic sequence CAAT(A/T)ATTG in their target genes (Ariel et al. 2007). To test if CaHDZ27 can bind this cis element, we performed chromatin immunoprecipitation (ChIP) analyses. We constructed two $\beta$-glucuronidase (GUS) reporter vectors (2xcis-p35Score::GUS and 2×mcis-p35Score::GUS), which were driven by a synthetic promoter containing two copies of the cis element (cis, CAATAATTG) or its mutant (mcis, CAGGGGTTG) and an adjacent Cauliflower mosaic virus (CaMV) 35S core promoter sequence. The control vector construct (p35Score::GUS) contained only the CaMV 35S core promoter sequence (Fig. 3A). The effect vector (35S:: CaHDZ27-HA) and reporter vectors were transformed into GV3101. Cells containing both effect and reporter vectors
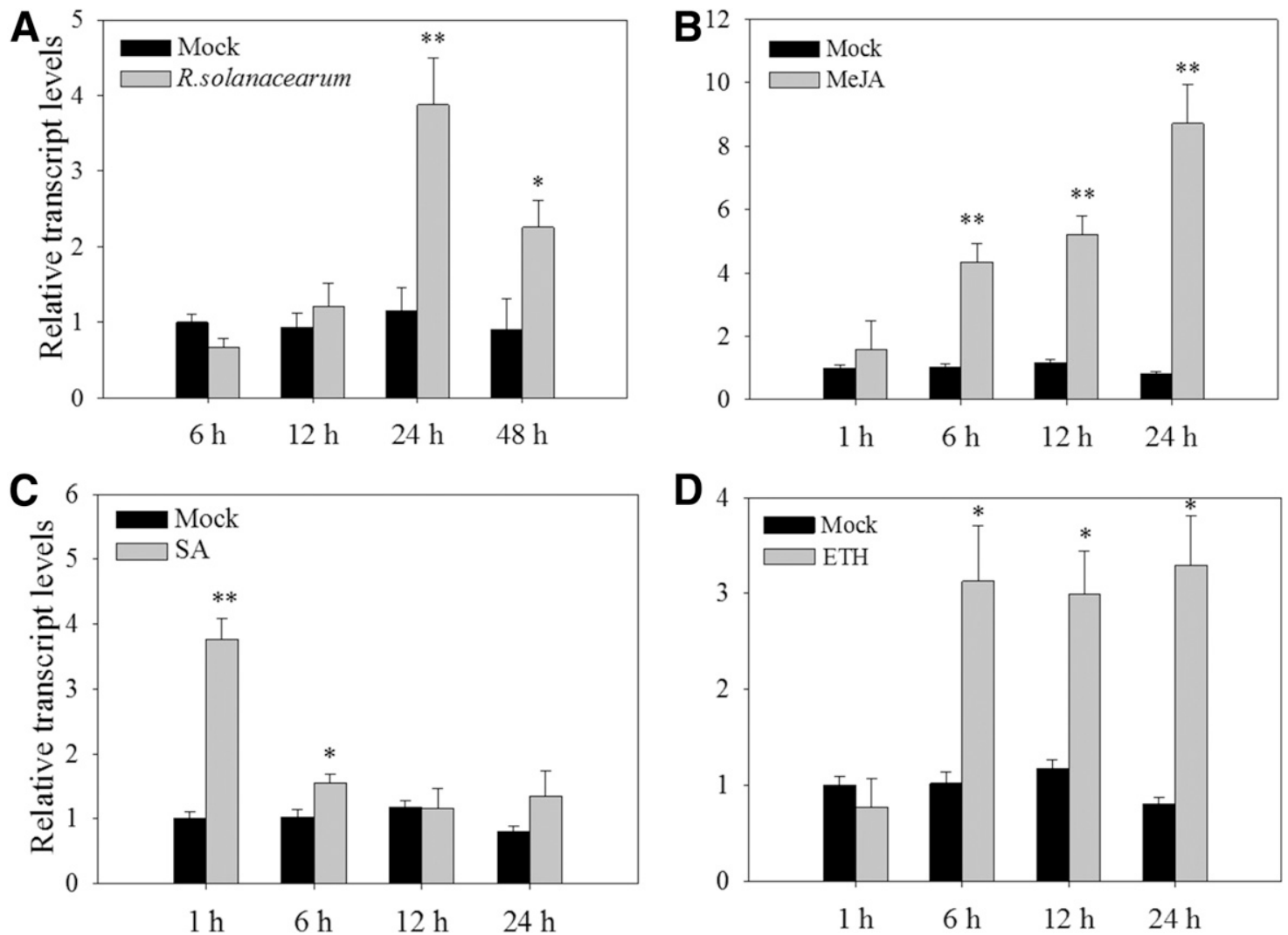

Fig. 2. Quantitative real time polymerase chain reaction analysis of relative CaHDZ27 transcript levels in pepper treated with Ralstonia solanacearum or exogenously applied phytohormones. A, CaHDZ27 transcript levels in pepper leaves were assessed at different timepoints after $R$. solanacearum inoculation. B to D, CaHDZ27 transcript levels were examined in pepper leaves at various timepoints after treatment with methyl jasmonate (MeJA, $100 \mu \mathrm{M})$, salicylic acid (SA, $1 \mathrm{mM})$, or ethephon $(\mathrm{ETH}, 100 \mu \mathrm{M})$. Error bars indicate standard error. Experiments were repeated three times along with at least three independent repetitions of the biological experiments. Asterisks ( $* *$ for $P<0.01$ and $*$ for $P<0.05$ indicate significant differences compared with the treatment and mock control, as determined by Student's $t$ test. 
were mixed at a $1: 1$ ratio and were infiltrated into pepper leaves, which were harvested after $48 \mathrm{~h}$ and were used for ChIP analyses. The results revealed that CaHDZ27 bound to the synthetic promoter containing the CAATAATTG cis element but did not bind to the promoter containing the mcis element (CAGGGGTTG) or only the CaMV $35 \mathrm{~S}$ core promoter sequence (Fig. 3B). We analyzed GUS activities in pepper leaves at 48 or $72 \mathrm{~h}$ after infiltration with GV3101 cells carrying effect and reporter vectors. The results showed that GUS activities were significantly higher after infiltration with $2 \times$ cis-p35Score::GUS compared with $2 \times$ m cis-p35Score::GUS or p35Score::GUS (Fig. 3C). These results indicate that CaHDZ27 can activate GUS expression by binding to the $c i$ s element (CAATAATTG).

\section{A Reporter vector}

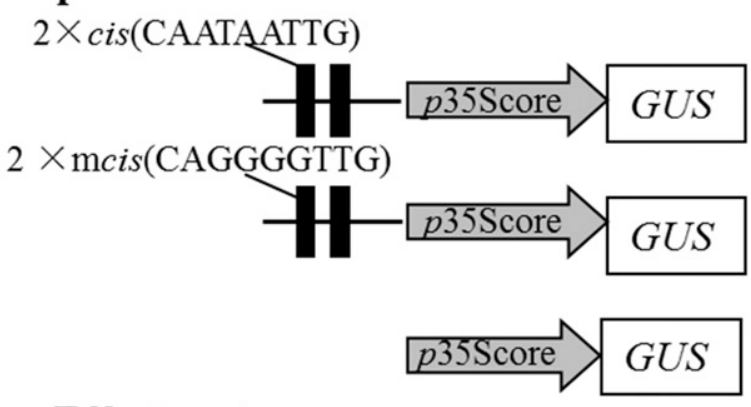

Effect vector

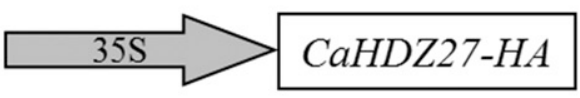

CaHDZ27 silencing significantly impairs pepper resistance against $R$. solanacearum infection.

The obtained results suggest that $C a H D Z 27$ might function as a TF and is upregulated by $R$. solanacearum infection. Therefore, we performed a gene silencing analysis using virus-induced gene silencing (VIGS) and a Tobacco rattle virus (TRV) vector containing a 185-bp fragment in the $3^{\prime}$ untranslated region (UTR) of CaHDZ27. Three independent experiments were performed, and each experiment included approximately 100 TRV::00 and 100 TRV::CaHDZ27 plants. The control plants expressed a pepper phytoene desaturase gene (TRV::CaPDS), which enabled visual inspection of gene silencing based on photobleaching (Supplementary Fig. S4). Reverse transcription (RT)-PCR demonstrated that the

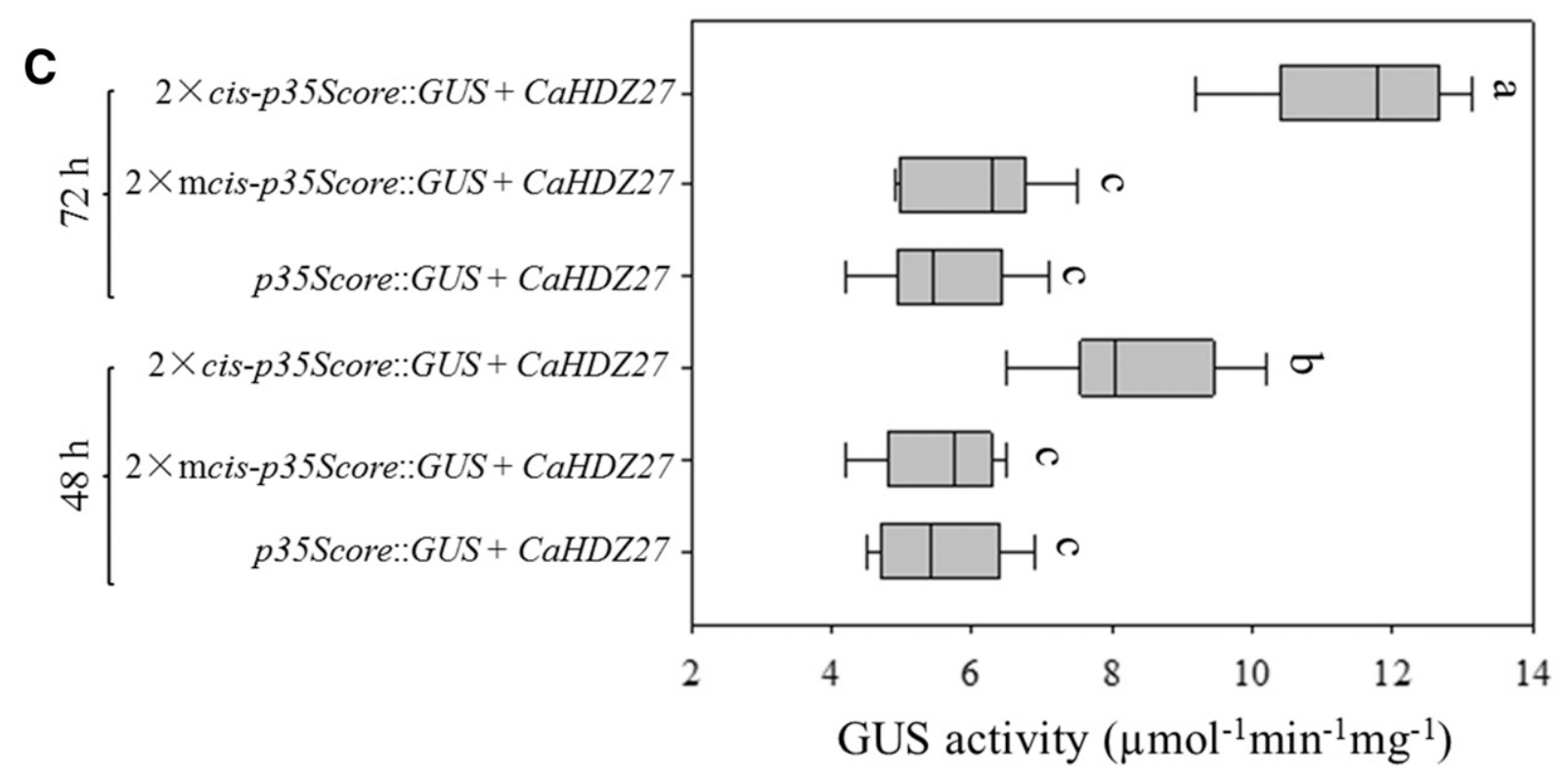

Fig. 3. CaHDZ27 binds the cis element (CAATAATTG) and triggers $\beta$-glucuronidase (GUS) expression in a CAATAATTG-dependent manner. A, Schematic diagram of the reporter and effect vectors used for the chromatin immunoprecipitation (ChIP) assay. The $2 \times c i s-p 35$ Score::GUS vector contains two tandem copies of the conserved HD-Zip I protein-binding cis element (CAATAATTG), whereas the $2 \times$ mcis-p35Score::GUS vector contains two tandem copies of mutant cis element (CAGGGGTTG), and p35Score::GUS contains only the Cauliflower mosaic virus 35S core promoter sequence. B, ChIP assay shows that CaHDZ27 binds the cis element (CAATAATTG). Agrobacterium tumefaciens GV3101 cells containing 35S::CaHDZ27-HA and 2×cis-p35Score::GUS, $2 \times$ mcis-p35Score::GUS, or p35Score::GUS constructs were coinfiltrated into pepper leaves, which were harvested after $48 \mathrm{~h}$ and were fixed with $1 \%$ formaldehyde. The input protein was immunoprecipitated with anti-hemagglutinin (anti-HA) antibodies. Acquired DNAs were adjusted to the same concentrations, and polymerase chain reaction-amplified using attB-specific primers. Input, total DNA-protein complex; ChIP (anti-HA), DNA-protein complex immunoprecipitated with anti-HA antibody. C, CaHDZ27 triggers GUS expression in a CAATAATTG-dependent manner. Pepper leaves were coinfiltrated with A. tumefaciens GV3101 cells carrying $2 \times$ cis-p35Score::GUS, $2 \times$ mcis-p35Score::GUS, p35Score::GUS, and 35S::CaHDZ27, and leaves were harvested after 48 or $72 \mathrm{~h}$. GUS activity was determined using a microplate reader. Bars represent medians, and boxes represent the 25th and 75 th percentiles. Different letters represent significant differences $(P<0.05$, least significant difference test). 
transcript level of CaHDZ27 is reduced in most of the TRV:: CaHDZ27-infiltrated plants, as compared with TRV::00-infiltrated plants (Supplementary Fig. S5). Subsequently, the CaHDZ27 silenced plants and TRV::00-infiltrated plants were inoculated by infiltrating leaves with an injection of $20 \mu \mathrm{l}$ of $R$. solanacearum FJC100301 cells $\left(10^{8} \mathrm{CFU} / \mathrm{ml}\right)$. The result of qPCR analysis showed that $\mathrm{CaHDZ27}$ transcript levels during $R$. solanacearum infection in TRV::CaHDZ27-infiltrated plants were significantly down-regulated compared with TRV::00 control plants (Fig. 4A).

Wounded root inoculations were performed by pouring $10 \mathrm{ml}$ of the $10^{8} \mathrm{CFU} / \mathrm{ml}$ suspensions into each pot. TRV::CaHDZ27 plants displayed leaf wilting at 10 days after root inoculation, and this progressed until complete leaf wilting was observed approximately 12 days after root inoculation. By contrast, plants inoculated with TRV::00 displayed delayed symptom development at 10 days after root inoculation (Fig. 4B; Supplementary Table S1) and symptom progression (leaf wilting) significantly differed in TRV::CaHDZ27 and control TRV::00 plants (Fig. 4C).

To investigate whether $C a H D Z 27$ silencing affects HRmediated cell death in response to $R$. solanacearum infection, we stained leaves of TRV:00 and TRV::CaHDZ27 plants with diaminobenzidine (DAB) (indicator of $\mathrm{H}_{2} \mathrm{O}_{2}$ accumulation) and trypan blue (indicator of cell death or necrosis) $48 \mathrm{~h}$ after $R$. solanacearum inoculation. The results showed that DAB and trypan blue staining intensities were higher in TRV::00 control leaves than in TRV::CaHDZ27 leaves (Fig. 4D). Consistently, electrolyte leakage levels were significantly higher in TRV:: 00 leaves than in TRV::CaHDZ27 leaves at 48 or $72 \mathrm{~h}$ after $R$. solanacearum infection (Fig. 4E). Notably, $\mathrm{H}_{2} \mathrm{O}_{2}$ accumulation and cell death during $R$. solanacearum infection were significantly attenuated in $\mathrm{CaHDZ27}$-silenced pepper leaves.

To confirm these phenotypic results and to investigate the mechanism of $\mathrm{CaHDZ27}$ activity in pepper immunity, we performed qPCR analysis of the following pepper defense-related gene expression levels in TRV::CaHDZ27 and TRV::00 control plants challenged with $R$. solanacearum: the HR marker gene CaHIRI (Choi et al. 2011), the ET biosynthesis-associated gene CaACOl (Cai et al. 2015), ET/JA-responsive gene CaPR4 (Cai et al. 2015), the reactive oxygen species (ROS) detoxification-associated gene $\mathrm{CaPO} 2$ (Choi et al. 2007), the SA-responsive gene CaPRl (Jung and Hwang 2000), and the basal defense-related gene CaBPRl (An et al. 2008). The results showed that transcript levels of CaHIR1, CaACO1, CaPR4, CaPO2, CaPR1, and CaBPR1 were significantly reduced in $C a H D Z 27$-silenced leaves compared with the levels in control plants with or without $R$. solanacearum infection (Fig. 4F). These combined results suggest that $\mathrm{CaHDZ27}$ silencing directly or indirectly downregulates the expression of these defenserelated genes during $R$. solanacearum infection, and enhances the susceptibility of pepper plants to $R$. solanacearum infection.

\section{Transient $\mathrm{CaHDZ27}$ expression triggers cell death and upregulates immunity-associated marker genes in pepper leaves.}

We performed gain-of-function analysis to further confirm the role of $\mathrm{CaHDZ27}$ in pepper immunity. For these experiments, we tested the transient expression of $\mathrm{CaHDZ27}$, using Agrobacterium infiltration into pepper leaves, and monitored CaHDZ27 expression by qPCR analysis. The results showed that $\mathrm{CaHDZ27}$ transcript levels were substantially higher in leaves transiently overexpressing CaHDZ27 (35S::CaHDZ27) compared with those in control leaves (35S::00) (Fig. 5A).

HR-mediated cell death is a hallmark indicator of ETI. To determine whether HR-mediated cell death is triggered by transient $C a H D Z 27$ overexpression, the infiltrated leaves were stained with trypan blue and DAB. The results showed that transient $\mathrm{CaHDZ27}$ overexpression resulted in more intense trypan blue and DAB staining, indicating that infiltrated leaves transiently overexpressing $\mathrm{CaHDZ27}$ have higher levels of cell death and $\mathrm{H}_{2} \mathrm{O}_{2}$ accumulation than control leaves $(35 \mathrm{~S}:: 00)$ (Fig. 5B). Higher levels of electrolyte leakage also occurred in leaves expressing $35 \mathrm{~S}:: \mathrm{CaHDZ} 27$ than in control leaves $(35 \mathrm{~S}:$ : 00 ) at 48 and $72 \mathrm{~h}$ after infiltration (Fig. 5C). We also performed a qPCR analysis of defense-marker gene expression, and the results showed higher expression levels of CaHIRl, CaACO1, CaPRl, CaPR4, CaPO2, and CaBPRl in leaves transiently expressing $\mathrm{CaHDZ27}$ than in control leaves (Fig. 5D). These combined results indicate that CaHDZ27 has a role in regulating HR- and defense-associated gene expression during pepper defense responses.

\section{Ectopic $\mathrm{CaHDZ27}$ overexpression confers}

\section{$R$. solanacearum resistance in transgenic tobacco.}

Stable transformation of pepper plants is still technically challenging. Therefore, we generated transformed tobacco (Nicotiana tabacum) plants overexpressing CaHDZ27, using A. tumefaciens-mediated leaf disc transformation. We acquired a number of homozygous $\mathrm{T}_{3}$ lines that did not exhibit significant morphological or developmental differences from wildtype plants. Three lines (\#3, \#5, and \#8) were randomly selected for further functional characterization of CaHDZ27 (Supplementary Fig. S6). By inoculating roots with $R$. solanacearum, leaf-wilting symptoms were observed in wild-type plants at 12 days after inoculation, whereas CaHDZ27-overexpressing transgenic plants (lines \#3, \#5, and \#8) exhibited only minimal leaf-wilting symptoms (Fig. 6A). The temporal progression of symptoms significantly differed between wild-type and CaHDZ27-overexpressing transgenic plants (Fig. 6B; Supplementary Table S2).

Next, we investigated $\mathrm{H}_{2} \mathrm{O}_{2}$ accumulation and HR-mediated cell death in response to $R$. solanacearum infiltration into leaf veins. After $48 \mathrm{~h}$, the infiltrated leaves were stained with DAB and trypan blue. The results showed that $\mathrm{H}_{2} \mathrm{O}_{2}$ accumulation and cell death were higher in the $\mathrm{CaHDZ27}$-overexpressing line \#3 challenged with $R$. solanacearum (Fig. 6C). Consistently, bacterial growth was significantly reduced in leaves of line \#3 compared with that in wild-type plants (Fig. 6D). These results indicate that $C a H D Z 27$ overexpression enhances the resistance of tobacco plants against $R$. solanacearum infection. The qPCR analysis indicated that transcript levels of HR marker genes (NtHSR201 [Sohn et al. 2007], ET biosynthesis-associated NtACC oxidase [Chen et al. 2003], and SA- or JA-associated NtPRa/c, NtPR2, NtPR3, and NtPR4 [Brogue et al. 1991; Ward et al. 1991; Dang et al. 2014]) were significantly higher in

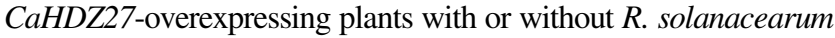
inoculation than in wild-type tobacco plants (Fig. 6E).

\section{CaHDZ27 can form homodimers.}

To test whether CaHDZ27 can form homodimers, we performed bimolecular fluorescence complementation (BiFC) and coimmunoprecipitation (CoIP) analyses. For the BiFC assay, CaHDZ27 was fused to the N- and C-terminal ends of yellow fluorescent protein (YFP) to generate CaHDZ27-nYFP and CaHDZ27-cYFP, respectively. Each construct was introduced into A. tumefaciens GV3101, and cells carrying different constructs were simultaneously infiltrated into $N$. benthamiana leaves. The infiltrated leaves were examined by confocal microscopy after $48 \mathrm{~h}$. YFP fluorescence was observed exclusively in nuclei in leaves infiltrated with both CaHDZ27-nYFP and CaHDZ27-cYFP, whereas no YFP fluorescence was observed in negative controls (coinfiltration of $n Y F P$ and $C a H D Z 27-c Y F P$, or $c Y F P$ and CaHDZ27-nYFP) (Fig. 7A). We also examined CaHDZ27 homodimerization using CoIP. FLAG-tagged CaHDZ27 and hemagglutinin (HA)-tagged CaHDZ27 were transiently coexpressed in $N$. benthamiana leaves by infiltration. After $48 \mathrm{~h}$, total protein was 
A
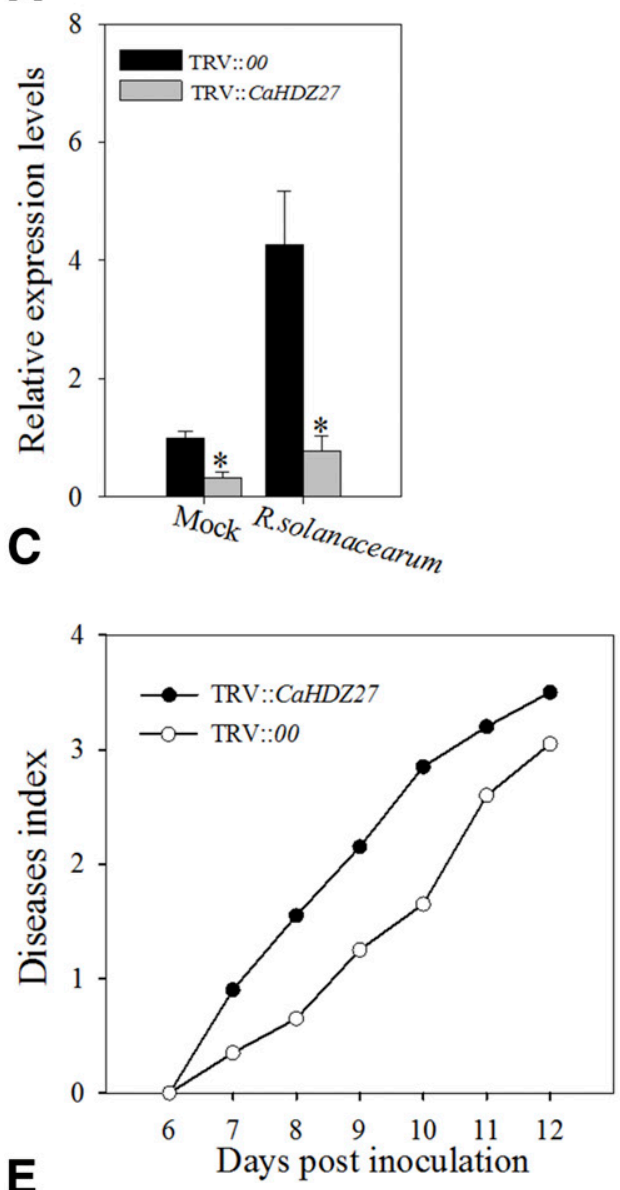

B

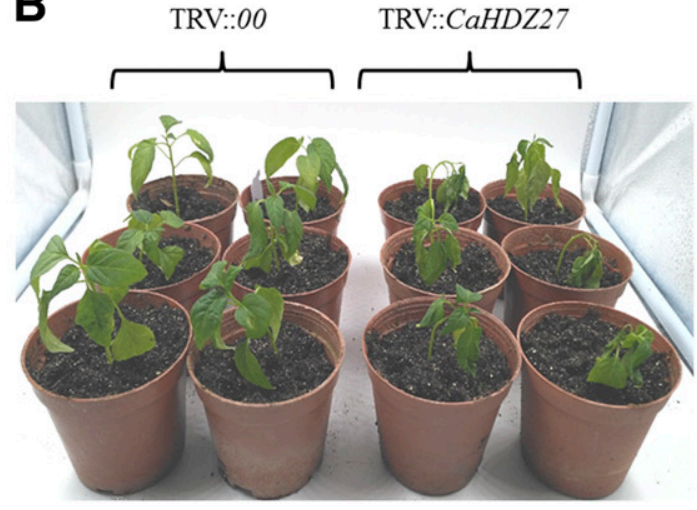

D

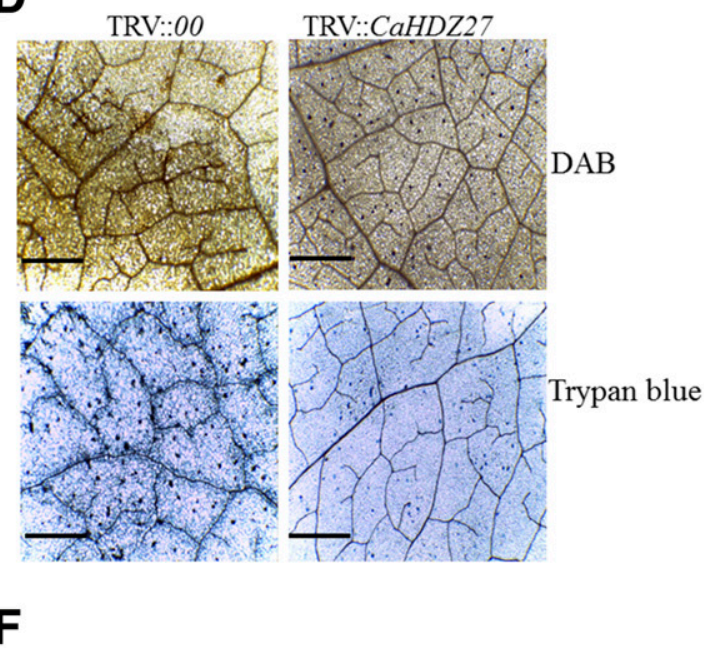

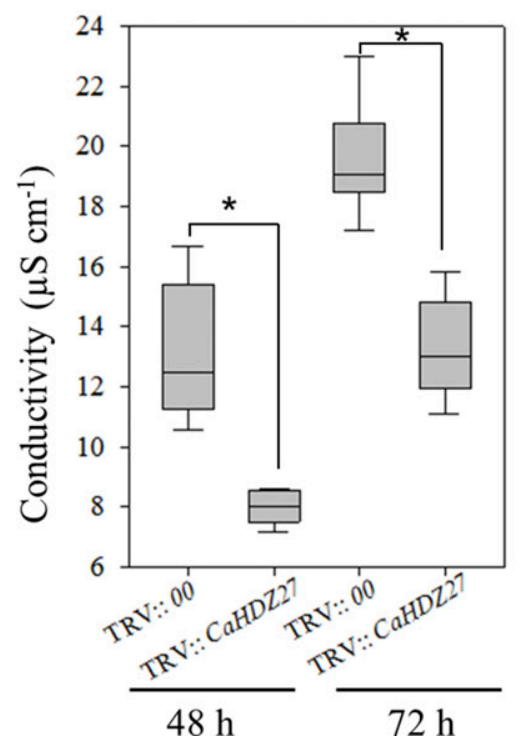

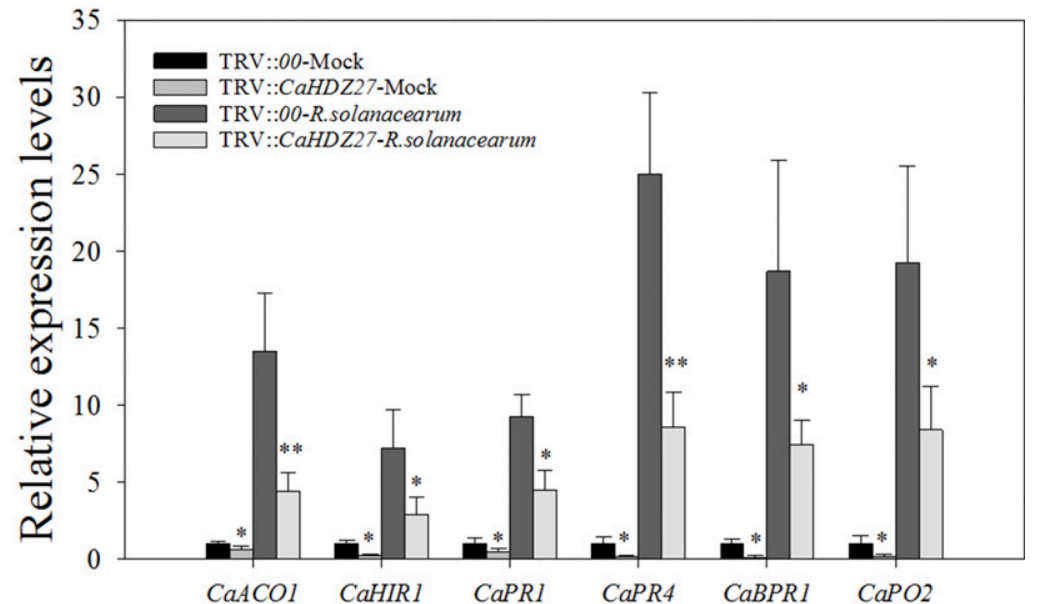

Fig. 4. Virus-induced gene silencing of CaHDZ27 in pepper plants enhances susceptibility to Ralstonia solanacearum infection. A, Quantitative polymerase chain reaction analysis of $C a H D Z 27$ transcript levels in TRV::00 or TRV::CaHDZ27 plants at $48 \mathrm{~h}$ after leaf inoculation with $R$. solanacearum. B, CaHDZ27 silencing in TRV::CaHDZ27 plants attenuated resistance to $R$. solanacearum infection. The wounded roots of pepper plants, silenced for 4 to 5 weeks, were inoculated with $R$. solanacearum. Several representative plants are shown at 10 days after inoculation. $\mathbf{C}$, Disease index score was recorded from 7 to 12 days after wounded root inoculation with $R$. solanacearum. Disease index score was determined according to the percentage of wilted leaves, with values defined as 0 (no symptoms), 1 ( 0 to $25 \%$ wilted leaves), 2 ( 25 to $50 \%$ wilted leaves), 3 (50 to $75 \%$ wilted leaves), and 4 (75 to $100 \%$ wilted leaves). Each point represents the mean disease index of 30 inoculated plants. The disease index score of TRV::CaHDZ27 plants was lower than TRV::00 plants throughout the entire experimental period. D, Visualization of diaminobenzidine (DAB) and trypan blue staining $48 \mathrm{~h}$ after leaf inoculation with $R$. solanacearum. The experiment was performed three times with similar results. Bars $=500 \mu \mathrm{m}$. E, Electrolyte leakage was measured in TRV::00 and TRV::CaHDZ27 leaves inoculated with $R$. solanacearum. Bars represent medians, and boxes represent the 25 th and 75 th percentiles. F, Transcript levels of defense-related genes were determined by quantitative reverse transcription-polymerase chain reaction in TRV::CaHDZ27 and TRV::00 pepper plants after inoculating leaves with $R$. solanacearum. Error bars show the mean of the standard deviation of replicate samples $(n=6)$. Asterisks (** for $P<0.01$ and $*$ for $P<0.05)$ indicate significant differences between TRV::00 and TRV::CaHDZ27 plants, as determined by Student's $t$ test. 
extracted and immunoprecipitated with FLAG-antibody-conjugated agarose beads, and the isolated proteins were then analyzed with HA antibody. The results show that CaHDZ27 can bind to itself to form homodimers (Fig. 7B).

\section{CaHDZ27 dimerization is enhanced} by $R$. solanacearum infection.

Next, we tested whether CaHDZ27 dimerization was affected by $R$. solanacearum infection by performing floating-leaf luciferase (LUC) complementation imaging using Gateway-compatible
split-LUC destination vectors (Gehl et al. 2011). We generated $35 S:: C a H D Z 27-N L U C, 35 S:: C a H D Z 27-C L U C$, and $p E X P-G U S$ (control) transient overexpression constructs and transformed them into A. tumefaciens GV3101 cells. Then, pepper leaves were coinfiltrated with the transient overexpression constructs. GUS activity was measured and used to normalize total protein levels, and dynamic protein-protein interactions were detected by luminescence activity measurement assays. The negative controls (coinfiltration of CaHDZ27-NLUC and CLUC, or CaHDZ27-CLUC and $N L U C)$ had lower luminescence activity than leaves coinfiltrated
A
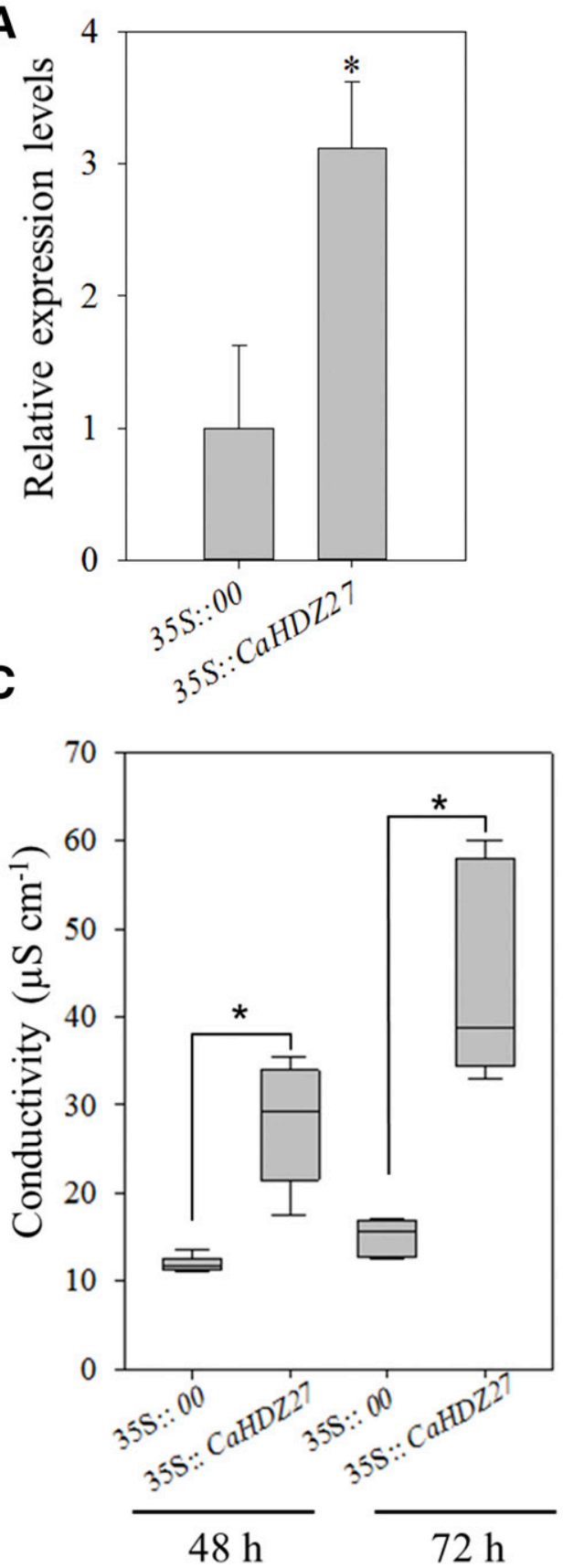

B

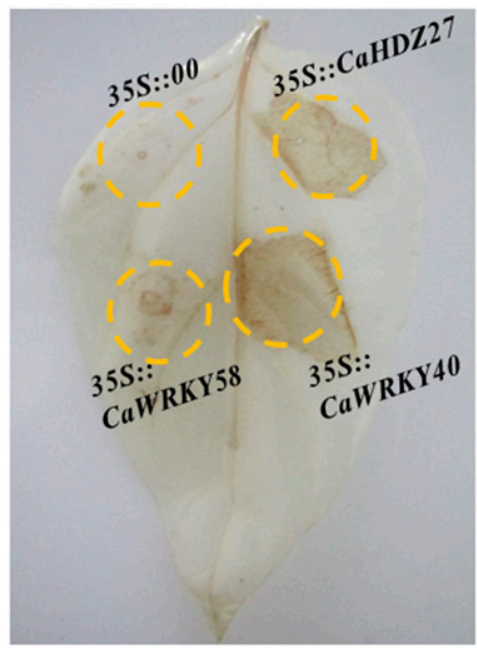

$\mathrm{DAB}$

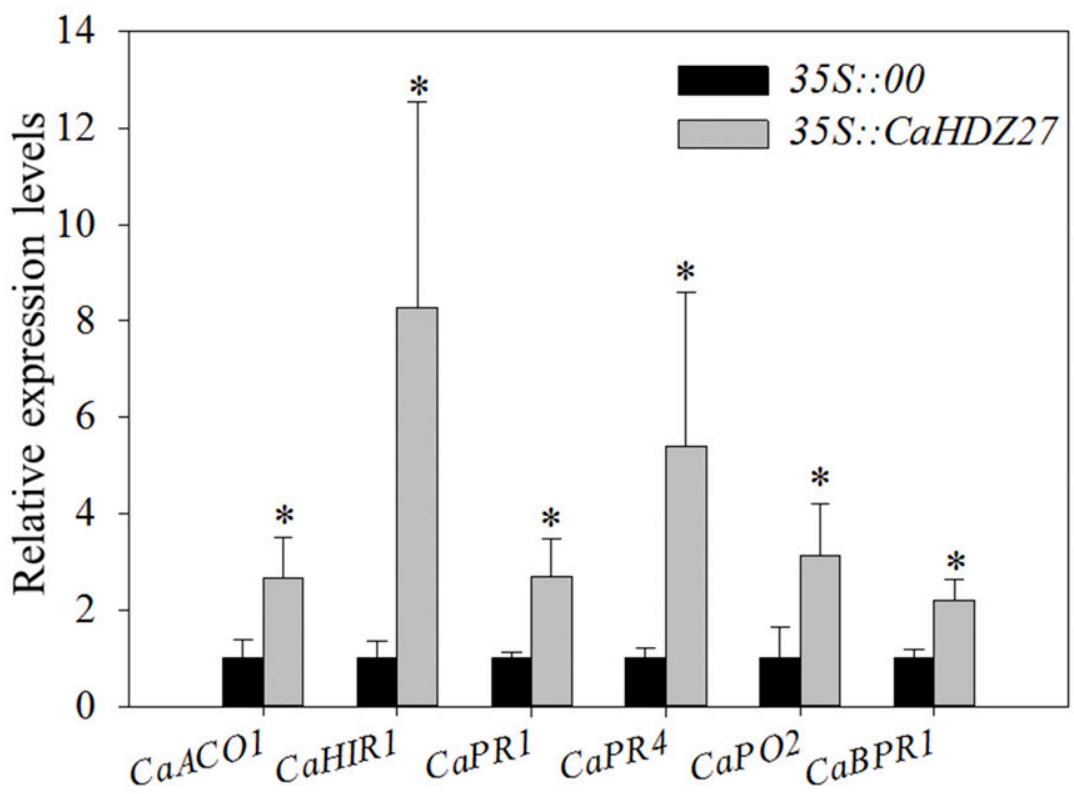

Fig. 5. Transient $\mathrm{CaHDZ27}$ overexpression in pepper leaves triggers immunity. A, Transient $\mathrm{CaHDZ27}$ overexpression was determined by quantitative reverse transcription-polymerase chain reaction analysis. B, Transient CaHDZ27 expression induces the cell-death response as visualized with diaminobenzidine (DAB) and trypan blue staining $48 \mathrm{~h}$ after infiltration. CaWRKY40 (Dang et al. 2013) and CaWRKY58 (Wang et al. 2013) were used as positive and negative controls, respectively. The experiment was performed three times with similar results. C, Quantification of electrolyte leakage to assess the cell-death response in pepper leaves transiently overexpressing CaHDZ27. Bars represent medians, and boxes represent the 25th and 75th percentiles. D, Transient CaHDZ27 overexpression upregulates the expression of defense-related marker genes compared with that in control plants at $48 \mathrm{~h}$ after infiltration. Data represent the mean \pm standard deviation $(n=6)$. One asterisk (* for $P<0.05$ ) indicates significant differences between $35 S:: 00$ and $35 S:: C a H D Z 27$ plants as determined by Student's $t$ test. 
A
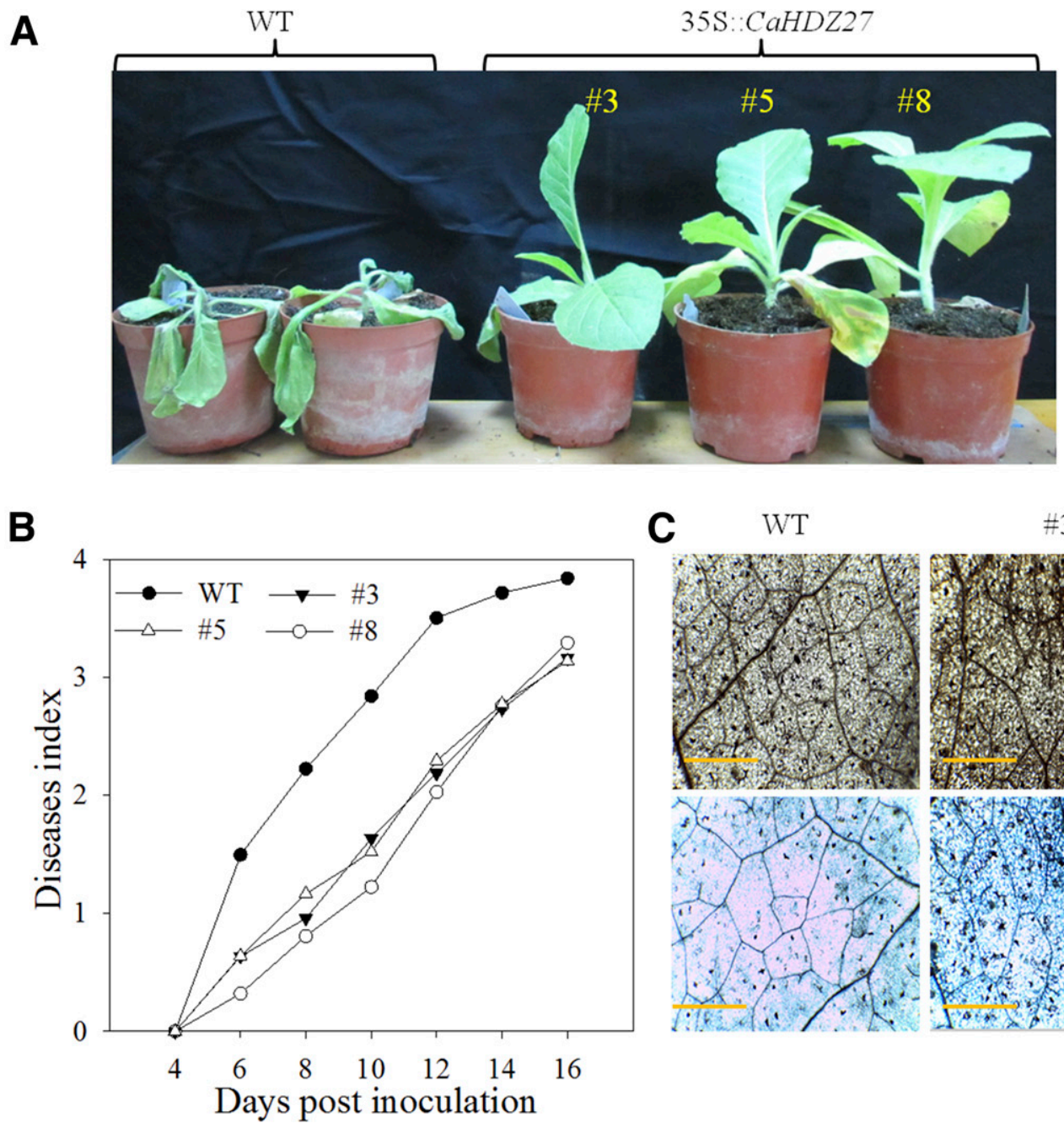

C WT
\#3

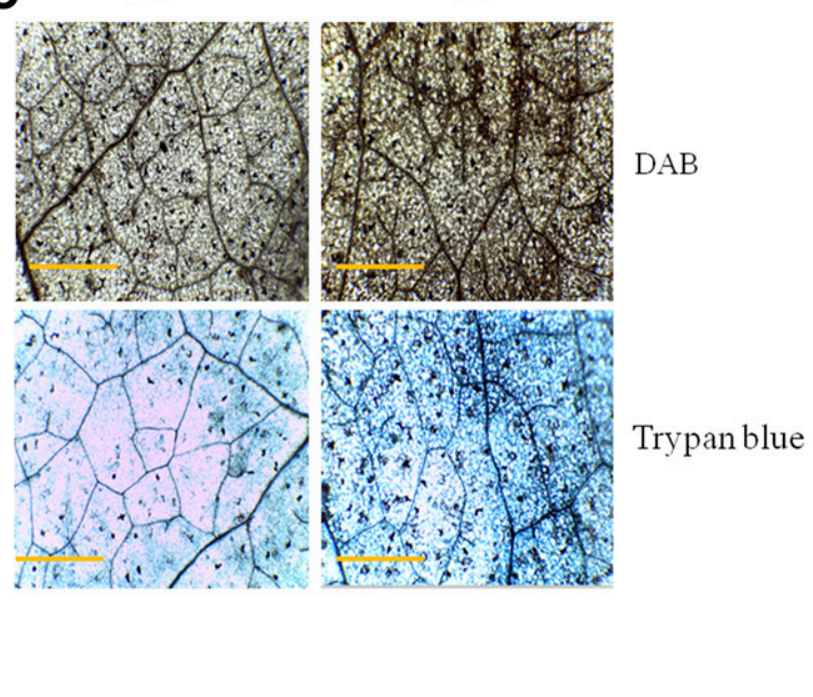

D

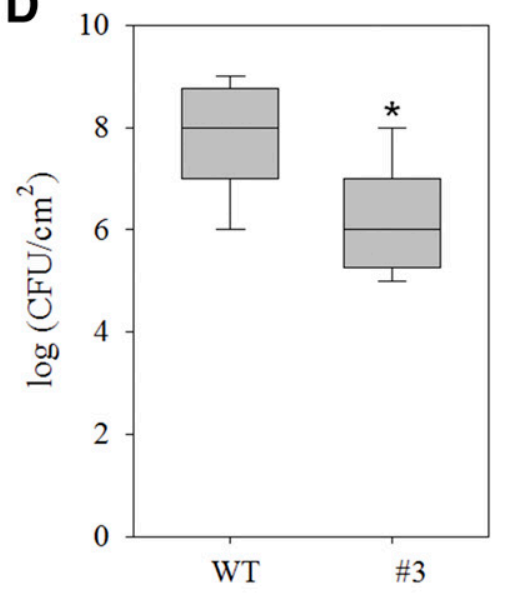

$\mathbf{E}$

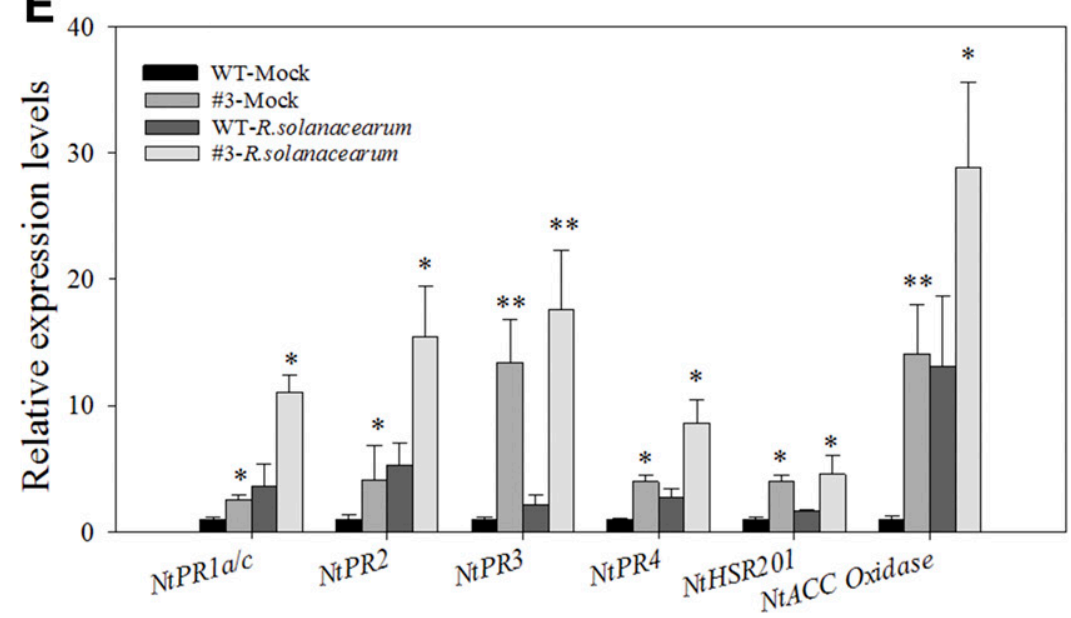

Fig. 6. Ectopic CaHDZ27 expression in tobacco plants enhances resistance against Ralstonia solanacearum infection. A, Transgenic CaHDZ27-overexpressing tobacco plants (6 weeks old) had higher resistance to $R$. solanacearum infection than wild-type (WT) plants at 12 days after inoculation of wounded roots. B, Disease index scores were recorded from wild-type and transgenic tobacco plants every 2 days from 6 to 16 days after inoculation of wounded roots with $R$. solanacearum. C, Hypersensitive response-mimicking cell death was visualized by diaminobenzidine (DAB) and trypan blue staining in transgenic CaHDZ27-overexpressing plants (line \#3) inoculated with $R$. solanacearum; DAB and trypan blue staining were more intense in CaHDZ27-overexpressing plants than in wild-type plants. Bars $=500 \mu \mathrm{m}$. D, Growth of $R$. solanacearum in transgenic CaHDZ27-overexpressing plants (line \#3) was significantly lower than in wild-type plants at $48 \mathrm{~h}$ after leaf inoculation. Bars represent medians, and boxes represent the 25 th and 75 th percentiles. E, Transcript levels of defenserelated genes were determined by quantitative polymerase chain reaction and were significantly higher in $C a H D Z 27-o v e r e x p r e s s i n g$ plants than in wild-type plants inoculated with $R$. solanacearum. Data represent the mean \pm standard deviation $(n=6)$. Asterisks $(* *$ for $P<0.01$ and $*$ for $P<0.05)$ indicate significant differences between wild-type and transgenic plants (line \#3) as determined by Student's $t$ test. 
with CaHDZ27-NLUC and CaHZ27-CLUC. These results suggest that CaHDZ27 can form homodimers. The luminescence activity in pepper leaves coinfiltrated with CaHDZ27-NLUC and CaHDZ27$C L U C$ was significantly increased at 1 day after $R$. solanacearum inoculation (Fig. 8), suggesting that CaHDZ27 homodimerization was enhanced by $R$. solanacearum infection.

\section{DISCUSSION}

HD-Zip proteins are unique TFs in plants, and HD-Zip I proteins have been implicated in abiotic stress responses (Harris et al. 2011; Zhang et al. 2014b; Zhao et al. 2014). Several HD-Zip I members have important roles in biotic stress responses, such as ATHB13 in Arabidopsis (Gao et al. 2014), H52 in tomato (Mayda et al. 1999), and HAHB4 in sunflower (Manavella et al. 2008). Information is lacking about pepper HD-Zip I members, and their biological functions are unknown. This study performed a functional genetic analysis of the CaHDZ27 HD-Zip I protein of Capsicum annuum. The results indicate that $\mathrm{CaHDZ27}$ functions as a positive regulator of $R$. solanacearum infection in pepper.

The deduced amino acid sequence of $\mathrm{CaHDZ27}$ contains a $\mathrm{HD}$ and an adjacent Zip domain and exhibits the highest sequence similarity with SIHZ27, among all HD-Zip I proteins in tomato and Arabidopsis. CaHDZ27 also has high sequence similarities with homologs from other species, including potato, tomato, and tobacco. We also show that CaHDZ27 binds to the 9-bp pseudopalindromic DNA sequence (CAATNATTG), which is specifically bound by HD-Zip class I proteins (Johannesson et al. 2001; Palena et al. 1999), and activates GUS expression in a CAATAATTG-dependent manner. These results suggest that CaHDZ27 is an HD-Zip class I protein in pepper. This conclusion was further supported by the observation that CaHDZ27-GFP expression was observed exclusively in the nuclei, which is frequently observed in other HD-Zip I proteins (Cao et al. 2016; Zhao et al. 2014).

Real-time qPCR analysis indicated that CaHDZ27 transcription was significantly induced by $R$. solanacearum inoculation, implying an involvement in pepper response to $R$. solanacearum. Plant genes that are upregulated in response to pathogen attack frequently have important roles in plant immunity (Cai et al. 2015; Dang et al. 2013, 2014; Shen et al. 2016a and b). Loss-of-function VIGS silencing experiments in pepper plants confirmed this hypothesis, because $\mathrm{CaHDZ27}$ silencing increased susceptibility to $R$. solanacearum infection and downregulated the defense-related marker genes CaHIRI (Choi et al. 2011, 2013; Jung and Hwang 2007; Jung et al. 2008), CaACO1 (Dong et al. 2014; Cheng et al. 2017), CaPRI (Yi et al. 2013; Kim et al. 2014), CaPR4 (Dong et al. 2014),

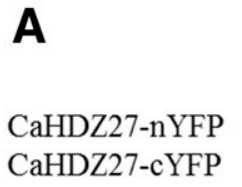

CaHDZ27-cYFP
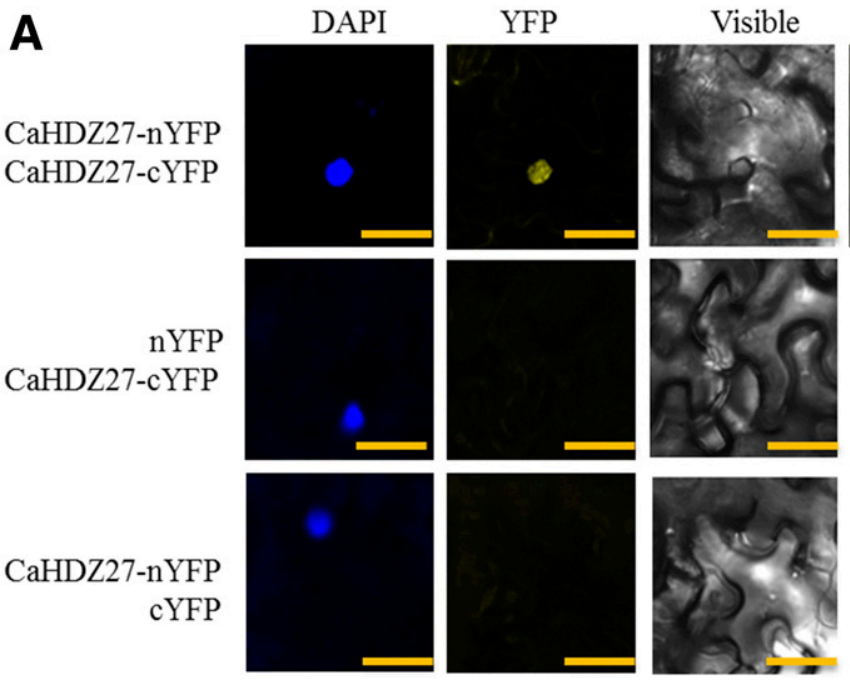
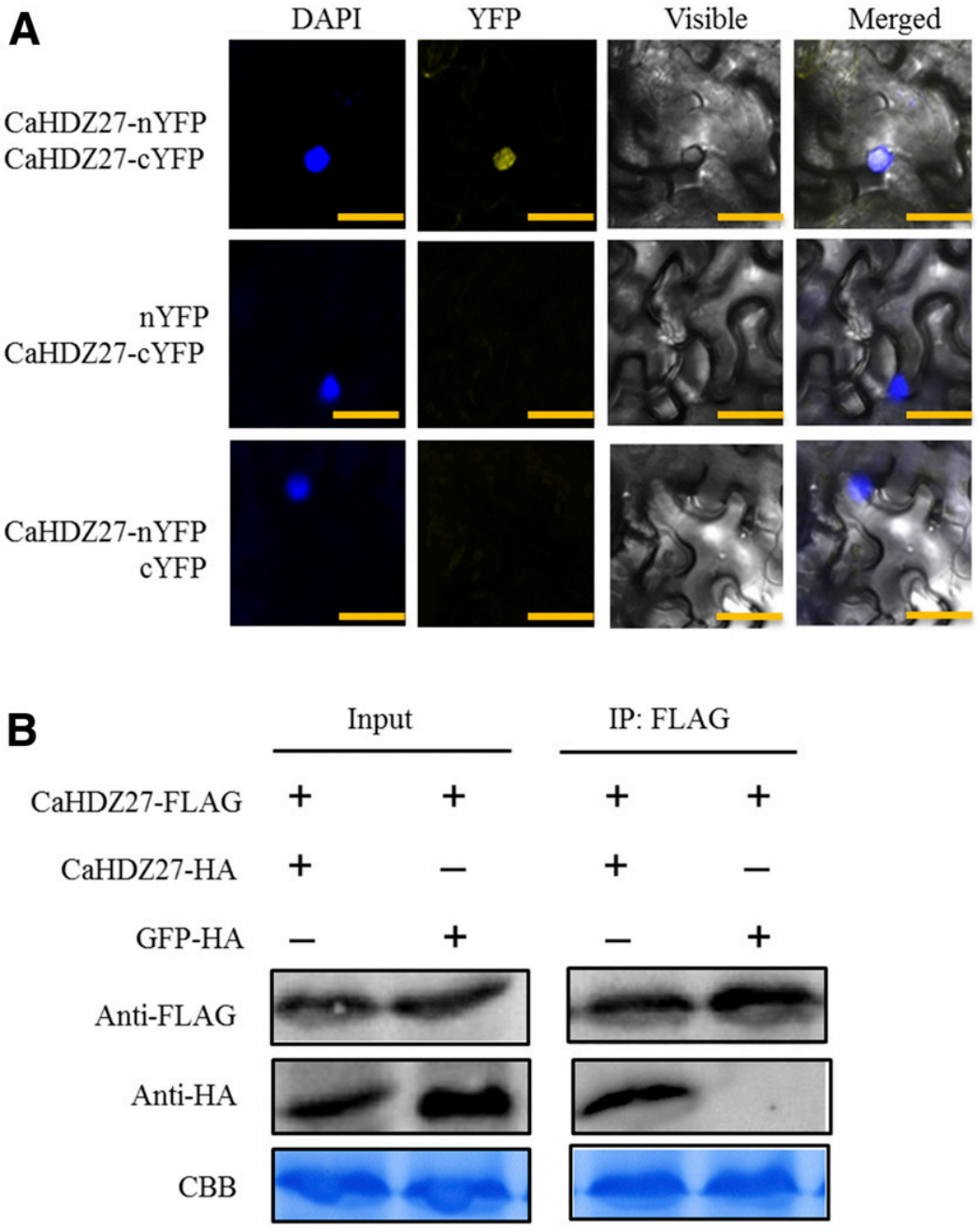

Fig. 7. Bimolecular fluorescence complementation (BiFC) and coimmunoprecipitation (Co-IP) assays indicate that CaHDZ27 binds to itself to form homodimers. A, BiFC analysis of Nicotiana benthamiana leaves transiently overexpressing CaHDZ27 indicate that CaHDZ27 forms homodimers. Bars $=$ $50 \mu \mathrm{m}$. B, Co-IP analysis of $N$. benthamiana leaves, transiently overexpressing CaHDZ27-FLAG and CaHDZ27-HA, confirms that CaHDZ27 forms homodimers. Leaves were harvested $48 \mathrm{~h}$ after infiltration, total protein was isolated, and soluble protein extracts before (input) and after immunoprecipitation (IP), with beads conjugated with anti-FLAG antibody, were detected by Western blotting with anti-HA (anti-hemagglutinin) antibody. 
CaPO2 (Choi and Hwang 2012; Choi et al. 2007), and CaBPR1 (Hong et al. 2005; Sarowar et al. 2005). By contrast, transient CaHDZ27 overexpression in pepper plants increased HRmimicking cell death and upregulated defense-related marker genes. Consistently, ectopic CaHDZ27 overexpression in transgenic tobacco enhanced resistance against $R$. solanacearum infection and induced $\mathrm{H}_{2} \mathrm{O}_{2}$ accumulation and defense-related gene expression of NtHSR201 (Czernic et al. 1996; Qiu et al. 2016; Takahashi et al. 2004), NtACC oxidase (Avni et al. 1994; Fammartino et al. 2010; Lasserre et al. 1997), NtPRla/c (Jamir et al. 2004; Matsuoka et al. 1987; Zhang et al. 2012b), NtPR2 (Ghanta et al. 2011; Naoumkina et al. 2008; Wang et al. 2013), NtPR3 (Zhang et al. 2012a), and NtPR4 (Li et al. 2015; Maimbo et al. 2007). These combined results indicate that $R$. solanacearum infection enhances CaHDZ27 expression and enhanced $\mathrm{CaHDZ27}$ expression in turn activates HR- or immunity-associated gene expression, leading to enhanced disease resistance.

The plant hormones SA, JA, and ET have key roles in regulating defense signaling networks that are activated in response to pathogen invasion (Pieterse et al. 2012). SA is primarily involved in biotrophic pathogen defense signaling, whereas JA and ET are primarily involved in necrotrophic pathogen defense signaling (Glazebrook 2005; Spoel et al. 2007). The production of SA, JA, and ET is frequently coupled with ETI or pathogentriggered immunity (PTI) (Tsuda and Katagiri 2010), and the defense phytohormone levels depend on the recognized pathogen (De Vos et al. 2005) and the invading population density (Pétriacq et al. 2016). The defense phytohormones can act synergistically or antagonistically, depending on their concentration levels (Kunkel and Brooks 2002; Mur et al. 2006). Recent work reported that SA and JA/ET are synergistically related in PTI but compensatorily related in ETI (Tsuda et al. 2009). The present study found that the $\mathrm{CaHDZ27}$ transcriptional response to exogenous application of MeJA, SA, and ETH was consistent with SA-, JA-, and ETresponsive pathogenesis-related marker gene expression in pepper and tobacco plants overexpressing $\mathrm{CaHDZ27}$. The expression of $\mathrm{CaPO}$, a marker gene for ROS-dependent resistance, also was upregulated by transient $\mathrm{CaHDZ27}$ overexpression in pepper.
These combined results indicate that $R$. solanacearum infection in pepper upregulates $\mathrm{CaHDZ27}$ expression, which in turn synergistically boosts SA-, JA-, and ET-mediated defense responses.

HD-Zip proteins form homodimers and heterodimers with other HD-Zip proteins in the same family (Johannesson et al. 2001; Meijer et al. 2000). Our results showed that CaHZ27 forms homodimers in pepper and this homodimeriztion was found to be enhanced by $R$. solanacearum infection, indicating that some unidentified signaling components might be activated by $R$. solanacearum, which potentiate homodimeriztion of CaHZ27. We speculate that the homodimeriztion of CaHZ27 might play a role in resistance of pepper to $R$. solanacearum infection; to confirm this speculation, further investigation and more evidence is required.

In summary, our data indicate that CaHDZ27 is a member of the HD-Zip I protein family in pepper. $\mathrm{CaHDZ27}$ is transcriptionally upregulated by $R$. solanacearum infection, thereby integrating SA-, JA-, and ET-dependent defense signaling. CaHDZ27 may act as a positive regulator of defense responses against $R$. solanacearum infection.

\section{MATERIALS AND METHODS}

Plant materials and growth conditions.

Seeds of pepper (Capsicum annuиm L. cv. Fj8) were obtained from the pepper breeding group in Fujian Agriculture and Forestry University (Fuzhou, Fujian, China). Tobacco (Nicotiana tabacum L. cv. Honghuadajinyuan) was supplied by the tobacco breeding group in Fujian Agriculture and Forestry University. Pepper and tobacco plants were grown in steam-sterilized soil mix in plastic pots at $25^{\circ} \mathrm{C}$ under a 16 -h day and 8 -h night photoperiod.

\section{Pathogens and inoculation procedures.}

Ralstonia solanacearum FJC100301 was cultured in potato sucrose agar (medium overnight at $28^{\circ} \mathrm{C}$, according to the method of Dang et al. (2013). The bacterial culture density was measured as $10^{8} \mathrm{CFU} / \mathrm{ml}$ (optical density at $600 \mathrm{~nm}\left[\mathrm{OD}_{600}\right]=0.8$ ) from serial dilutions in $10 \mathrm{mM} \mathrm{MgCl} 2$. Wounded root inoculation was

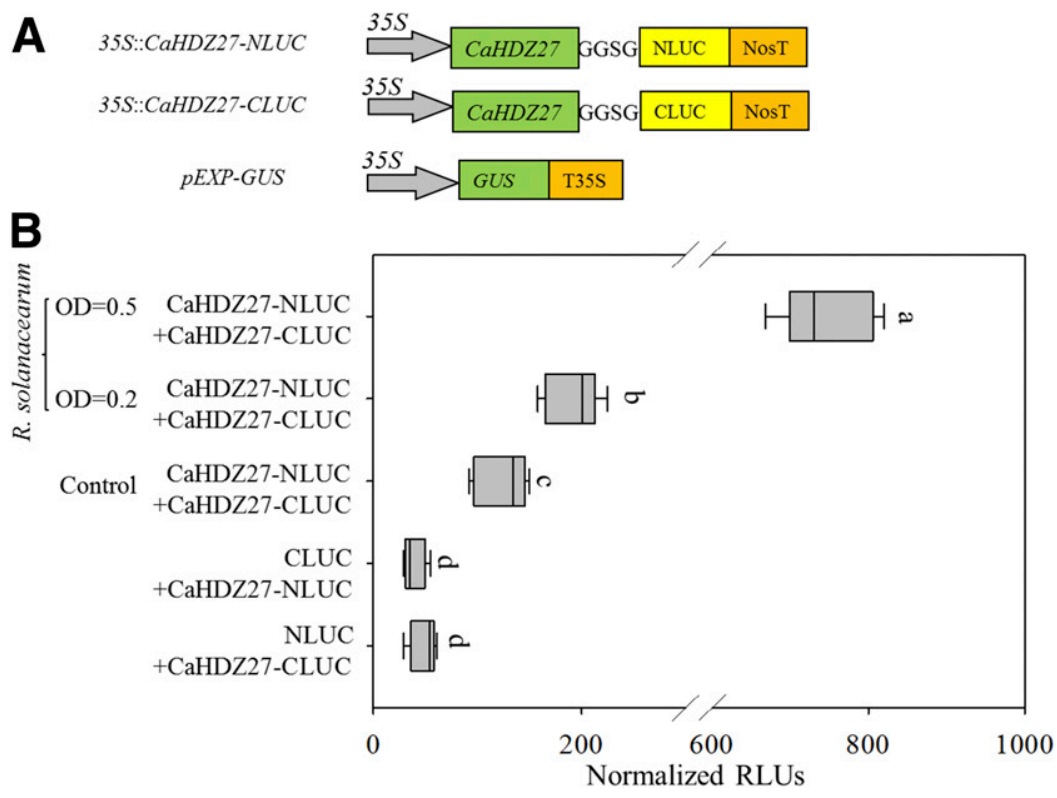

Fig. 8. Luminescence activity measurement assay to quantify the strength of CaHDZ27 homodimerization in pepper leaves inoculated with Ralstonia solanacearum. A, Schematic representation of 35S::CaHDZ27-NLUC and 35S::CaHDZ27-CLUC constructs for analysis of CaHDZ27 homodimerization. The pEXP-GUS construct was used for $\beta$-glucuronidase activity measurement, which was used to normalize the total protein concentration. B, Luminescence activity measurement analysis of CaHDZ27 homodimers in pepper leaves responding to R. solanacearum inoculation. Different letters represent significant differences as determined by the least significant difference test $(P<0.05)$. Bars represent medians, and boxes represent the 25 th and 75 th percentiles. 
performed by pouring $10 \mathrm{ml}$ of the $10^{8}-\mathrm{CFU} / \mathrm{ml}$ bacterial suspension into each pot. Before inoculation, roots were slightly damaged by making three holes in the soil. Then, the pots were kept in a growth room at $28^{\circ} \mathrm{C}$ with soil moisture $>90 \%$.

For RNA extraction, trypan blue and DAB staining, and ion conductivity experiments, plants were inoculated by infiltrating bacterial suspension $\left(\mathrm{OD}_{600}=0.8\right)$ into the third leaf from the apical meristem, using a syringe without a needle, and mock control plants were inoculated with sterile $10 \mathrm{mM} \mathrm{MgCl}_{2}$.

\section{Treatment of plants with exogenous hormones.}

Pepper plants at the six-leaf stage were used to investigate CaHDZ27 expression patterns in response to exogenous application of hormones or growth regulators. Plants were sprayed with $0.1,1$, or $10 \mathrm{mM} \mathrm{SA}$ (dissolved in $10 \%$ ethanol), 10,100 , or $1,000 \mu \mathrm{M}$ MeJA (dissolved in $10 \%$ ethanol), or 10,100 , or $1,000 \mu \mathrm{M} \mathrm{ETH}$ (dissolved in sterile distilled and deionized $\mathrm{H}_{2} \mathrm{O}$ ). Mock control plants were sprayed with $10 \%$ ethanol or sterile distilled and deionized $\mathrm{H}_{2} \mathrm{O}$.

\section{Vector construction.}

Vectors were constructed using a Gateway cloning technique (Invitrogen, Carlsbad, CA, U.S.A.), and a series of Gatewaycompatible destination vectors were employed. The ORF of CaHDZ27, with or without the stop codon, was initially amplified by PCR with its specific primer pair (5'-ATGGAAGTTG GAGGAGTTATT-3' and 5'-CTATTGCCAGAACCAACAAGG$3^{\prime}$ ) flanked with attB for Gateway cloning and GXL DNA polymerase (Takara, Osaka, Japan). The amplified product was confirmed by sequencing. The $\mathrm{CaHDZ27} \mathrm{ORF}$ was cloned into the entry vector pDONR207, using the BP reaction and was then cloned into destination vectors such as pMDC83, pK7WG2, and pEarleyGate201, using the LR reaction for subcellularr localization, transient overexpression, or ChIP analysis, respectively.

DNA fragments, containing two copies of conserved HD-Zip I protein-binding cis element (CAATAATTG), or two copies of mutant element (CAGGGGTTG) and the 54-bp minimal CaMV 35S promoter flanked with $a t t B$, were synthesized by Convenience Corporation (Suzhou, China). These were cloned into the pMDC163 destination vector by sequential BP and LR reactions for promoter expression assays of the GUS reporter gene in pepper plants.

To construct the gene-silencing vectors for VIGS analysis, a fragment (approximately $185 \mathrm{bp}$ ) of the 3' UTR of CaHDZ27 was amplified by PCR with a specific primer pair (5'-TTCCA CAAGAGAATAGTG-3' and 5'-GGAACAAAGCTAATAAA-3'). The fragment was cloned into the VIGS vector TRV2 (PYL279), using the Gateway cloning technique, as described above.

For construction of the BiFC vector, the $C a H D Z 27$ ORF without the stop codon was cloned into pDONR207, using the BP reaction. Then, the LR reaction was used to recombine the entry plasmids into the Gateway binary destination vectors puc-SPYNE ${ }^{\mathrm{GW}}$ and puc-SPYCE $^{\mathrm{GW}}$, which contain the N-terminal or C-terminal fragment of YFP (nYFP or cYFP, respectively). Thus, the CaHDZ27 protein was independently tagged with $n Y F P$ or cYFP at either the $\mathrm{N}$ or $\mathrm{C}$ terminus.

Split-LUC vectors were constructed by amplifying the $a t t B$ flanked $\mathrm{CaHDZ27} \mathrm{ORF}$ without stop codon, using genespecific primers. The PCR products were introduced into the satellite vector pDONR207. The CaHDZ27 entry vector was transformed into the destination vectors (pDEST- ${ }^{\mathrm{GW}} \mathrm{NLUC}$ and pDEST- ${ }^{\mathrm{GW}}$ CLUC) to generate $35 \mathrm{~S}:: \mathrm{CaHDZ27-NLUC}$ and $35 \mathrm{~S}::$ CaHDZ27-CLUC, respectively.

\section{Transient expression of $\mathrm{CaHDZ27}$ in pepper leaves.}

The 35S::CaHDZ27 vector was transformed into A. tumefaciens GV3101. GV3101 cells carrying the $35 S:: C a H D Z 27$ vector were grown overnight in YEP medium with appropriate antibiotics. Cells were suspended in infiltration buffer (10 mM MES, pH 5.7, $10 \mathrm{mM} \mathrm{MgCl}$, and $200 \mu \mathrm{M}$ acetosyringone). The cell suspension $\left(\mathrm{OD}_{600}=0.8\right)$ was infiltrated into leaves of pepper plants at the eight-leaf stage, using a syringe without a needle. The plants were kept in a growth room at $25^{\circ} \mathrm{C}, 60$ to $70 \mathrm{mmol}$ photons $/ \mathrm{m} / \mathrm{s}$, $70 \%$ relative humidity, and a 16-h light and 8-h dark photoperiod, for 2 days before injected leaves were used for assays.

\section{Tobacco transformation.}

Transgenic tobacco plants overexpressing $\mathrm{CaHDZ27}$ were generated using Agrobacterium-mediated tobacco leaf disc transformation (Oh et al. 2005). Eight kanamycin-resistant lines of transgenic tobacco plants harboring the $35 \mathrm{~S}:: \mathrm{CaHDZ27}$ construct were selected and were confirmed with RT-PCR analysis. Seeds of $\mathrm{T}_{1}$ plants were collected from regenerated $\mathrm{T}_{0}$ plants, and seedlings of $T_{2}$ and $T_{3}$ lines were further selected on Murashige-Skoog agar plates containing $50 \mu \mathrm{g}$ of kanamycin per milliliter. $\mathrm{T}_{3}$ plants were used for analyses.

\section{Subcellular localization of CaHDZ27.}

The 35S::CaHDZ27-GFP and 35S::GFP constructs were transformed into A. tumefaciens GV3101 and were grown overnight. Then, GV3101 cells were suspended in infiltration buffer $(10 \mathrm{mM}$ MES, pH 5.7, $10 \mathrm{mM} \mathrm{MgCl}$, and $200 \mu \mathrm{M}$ acetosyringone) and were infiltrated into $N$. benthamiana leaves, using a syringe without a needle. For DAPI staining, $N$. benthamiana leaves were immersed in DAPI solution ( $0.1 \%$ DAPI and 5\% dimethyl sulfoxide [DMSO]) and were incubated at $37^{\circ} \mathrm{C}$ for $1 \mathrm{~h}$, before observation. GFP fluorescence was imaged using a laser scanning confocal microscope (TCS SP8; Leica, Solms, Germany) with an excitation wavelength of $488 \mathrm{~nm}$ and a 505 to $530 \mathrm{~nm}$ band-pass emission filter.

\section{VIGS of $\mathrm{CaHDZ27}$ in pepper plants.}

VIGS of CaHDZ27 was performed according to a previously described method (Dang et al. 2013). GV3101 cells harboring TRV1 (PYL192) with TRV2 (PYL279) and TRV2::CaHDZ27 $\left(\mathrm{OD}_{600}=\right.$ 0.8 ) were mixed at a 1:1 ratio, and the mixtures were coinfiltrated into cotyledons of 2-week-old pepper seedlings. The seedlings were incubated at $16^{\circ} \mathrm{C}$ for $56 \mathrm{~h}$ and were then grown at $25^{\circ} \mathrm{C}$.

\section{Histochemical staining and ion conductivity measurement assay.}

Histochemical staining and ion conductivity measurements were conducted using previously published methods (Choi et al. 2012). Agrobacterium-infiltrated leaves were stained with DAB for $\mathrm{H}_{2} \mathrm{O}_{2}$ detection and with trypan blue for cell-death detection. To quantify cell death in pepper leaves, ion conductivity was measured, using a Mettler Toledo 326 (Mettler, Zurich, Switzerland), as described previously (Cai et al. 2015).

\section{Real-time qPCR.}

The qPCR analysis was performed, using a Bio-Rad real-time PCR system (Foster City, CA, U.S.A.) and the SYBR Premix Ex Taq II system (Takara), as described previously (Cai et al. 2015; Dang et al. 2013). The expression of CaHDZ27 and defense-related genes in pepper or tobacco plants were examined, using the primers listed in Supplementary Tables S3 and S4. Relative transcript levels were normalized with respect to the levels of CaActin and 18srRNA in pepper or NtActin and NtEEl $\alpha$ in tobacco. Data were calculated by the by the $2^{-\Delta \Delta C T}$ method, as described previously (Livak and Schmittgen 2001). In each case, three technical replicates were performed for at least three independent biological replicates.

\section{ChIP analysis.}

ChIP assays were performed as described previously (Cai et al. 2015). The leaves of pepper plants at the eight-leaf stage were 
infiltrated with $A$. tumefaciens GV3101 cells carrying the effect vector (35S::CaHDZ27-HA) and reporter vectors (2xcis-p35Score:: GUS, 2xmcis-p35Score::GUS, and p35Score::GUS). T-DNA insertion into the pepper genome was confirmed. After $48 \mathrm{~h}$, approximately $2 \mathrm{~g}$ of leaves were harvested and treated with either $10 \mathrm{mM}$ bithionol sulfoxide or DMSO (solvent control) for $16 \mathrm{~h}$ and were then fixed with $1.0 \%$ formaldehyde for $5 \mathrm{~min}$. The chromatin was isolated and sheared to an average length of 500 bp. Chromatin fragments that bound to CaHDZ27 were immunoprecipitated using the HA antibody (Santa Cruz Biotechnology, Santa Cruz, CA, U.S.A.). The resulting DNA fragments were isolated and were used as templates for PCR analysis with $a t t B$-specific primer pairs.

\section{BiFC analysis.}

BiFC was performed as described previously (Liu et al. 2016). CaHDZ27-nYFP and CaHDZ27-cYFP were introduced into A. tumefaciens $\mathrm{GV} 3101$, and cells carrying the BiFC constructs were infiltrated into $N$. benthamiana leaves. After $48 \mathrm{~h}$, leaf fluorescence was analyzed using a confocal microscope.

\section{CoIP assay.}

The 35S::CaHDZ27-FLAG and 35S::CaHDZ27-HA constructs were transformed into A. tumefaciens GV3101, and cells carrying $35 S:: C a H D Z 27-F L A G$ or $35 S:: C a H D Z 27-H A$ were coinfiltrated into $N$. benthamiana leaves at a ratio of $1: 1$. The Co-IP assay was performed as described previously (Liu et al. 2016).

\section{LUC activity determination.}

GV3101 cells carrying the appropriate split-LUC constructs were to infiltrate pepper leaves. For coexpression analyses, cells carrying the different vectors were mixed before infiltration. GV3101 cells carrying the appropriate split-LUC constructs were infiltrated by injecting 5-week-old pepper leaves. After 1 day, the infiltrated leaves were inoculated with $20 \mu \mathrm{l}$ of $R$. solanacearum cells, and leaves were harvested for LUC activity assay after 1 day. LUC activity reconstituted from the $35 S:: C a H D Z 27-N L U C$ and $35 S:: C a H D Z 27-C L U C$ vectors was determined as described previously (Liu et al. 2016).

\section{ACKNOWLEDGMENTS}

We thank M. D. Curtis for kindly providing the Gateway destination vectors and S. P. Dinesh-Kumar (Yale University) for the pTRV1 and pTRV2 vectors. This work was supported by grants from the National Natural Science Foundation of China (31301254, 31372061, 31401890, 31401312, 31260482, and 31060263)

\section{LITERATURE CITED}

AbuQamar, S., Chen, X., Dhawan, R., Bluhm, B., Salmeron, J., Lam, S., Dietrich, R. A., and Mengiste, T. 2006. Expression profiling and mutant analysis reveals complex regulatory networks involved in Arabidopsis response to Botrytis infection. Plant J. 48:28-44.

An, S. H., Choi, H. W., Hwang, I. S., Hong, J. K., and Hwang, B. K. 2008. A novel pepper membrane-located receptor-like protein gene CaMRP1 is required for disease susceptibility, methyl jasmonate insensitivity and salt tolerance. Plant Mol. Biol. 67:519-533.

Ariel, F. D., Manavella, P. A., Dezar, C. A., and Chan, R. L. 2007. The true story of the HD-Zip family. Trends Plant Sci. 12:419-426.

Avni, A., Bailey, B. A., Mattoo, A. K., and Anderson, J. D. 1994. Induction of ethylene biosynthesis in Nicotiana tabacum by a Trichoderma viride xylanase is correlated to the accumulation of 1-aminocyclopropane-1carboxylic acid (ACC) synthase and ACC oxidase transcripts. Plant Physiol. 106:1049-1055.

Brandt, R., Cabedo, M., Xie, Y., and Wenkel, S. 2014. Homeodomain leucine-zipper proteins and their role in synchronizing growth and development with the environment. J. Integr. Plant Biol. 56:518-526.

Brogue, K., Chet, I., Holliday, M., Cressman, R., Biddle, P., Knowlton, S. Mauvais, C. J., and Broglie, R. 1991. Transgenic plants with enhanced resistance to the fungal pathogen Rhizoctonia solani. Science 254: 1194-1197.

Cabello, J. V., Giacomelli, J. I., Piattoni, C. V., Iglesias, A. A., and Chan, R. L. 2016. The sunflower transcription factor HaHB11 improves yield, biomass and tolerance to flooding in transgenic Arabidopsis plants J. Biotechnol. 222:73-83.

Cai, H., Yang, S., Yan, Y., Xiao, Z., Cheng, J., Wu, J., Qiu, A., Lai, Y., Mou, S., Guan, D., Huang, R., and He, S. 2015. CaWRKY6 transcriptionally activates CaWRKY4O, regulates Ralstonia solanacearum resistance, and confers high-temperature and high-humidity tolerance in pepper. J. Exp. Bot. 66:3163-3174.

Cao, L., Yu, Y., DuanMu, H., Chen, C., Duan, X., Zhu, P., Chen, R., Li, Q., Zhu, Y., and Ding, X. 2016. A novel Glycine soja homeodomain-leucine zipper (HD-Zip) I gene, Gshdz4, positively regulates bicarbonate tolerance and responds to osmotic stress in Arabidopsis. BMC Plant Biol. 16:184.

Chen, N., Goodwin, P. H., and Hsiang, T. 2003. The role of ethylene during the infection of Nicotiana tabacum by Colletotrichum destructivum. J. Exp. Bot. 54:2449-2456.

Chen, X., Chen, Z., Zhao, H., Zhao, Y., Cheng, B., and Xiang, Y. 2014. Genome-wide analysis of soybean HD-Zip gene family and expression profiling under salinity and drought treatments. PLoS One 9:e87156.

Cheng, W., Xiao, Z., Cai, H., Wang, C., Hu, Y., Xiao, Y., Zheng, Y., Shen, L., Yang, S., Liu, Z., Mou, S., Qiu, A., Guan, D., and He, S. 2017. A novel leucine-rich repeat protein, CaLRR51, acts as a positive regulator in the response of pepper to Ralstonia solanacearum infection. Mol. Plant Pathol. 18:1089-1100.

Choi, D. S., Hwang, I. S., and Hwang, B. K. 2012. Requirement of the cytosolic interaction between PATHOGENESIS-RELATED PROTEIN10 and LEUCINE-RICH REPEAT PROTEIN1 for cell death and defense signaling in pepper. Plant Cell 24:1675-1690.

Choi, H. W., and Hwang, B. K. 2012. The pepper extracellular peroxidase $\mathrm{CaPO} 2$ is required for salt, drought and oxidative stress tolerance as well as resistance to fungal pathogens. Planta 235:1369-1382.

Choi, H. W., Kim, D. S., Kim, N. H., Jung, H. W., Ham, J. H., and Hwang, B. K. 2013. Xanthomonas filamentous hemagglutinin-like protein Fha1 interacts with pepper hypersensitive-induced reaction protein CaHIR1 and functions as a virulence factor in host plants. Mol. Plant-Microbe Interact 26:1441-1454.

Choi, H. W., Kim, Y. J., and Hwang, B. K. 2011. The hypersensitive induced reaction and leucine-rich repeat proteins regulate plant cell death associated with disease and plant immunity. Mol. Plant-Microbe Interact 24:68-78.

Choi, H. W., Kim, Y. J., Lee, S. C., Hong, J. K., and Hwang, B. K. 2007. Hydrogen peroxide generation by the pepper extracellular peroxidase $\mathrm{CaPO} 2$ activates local and systemic cell death and defense response to bacterial pathogens. Plant Physiol. 145:890-904.

Czernic, P., Huang, H. C., and Marco, Y. 1996. Characterization of hsr201 and $h s r 515$, two tobacco genes preferentially expressed during the hypersensitive reaction provoked by phytopathogenic bacteria. Plant Mol. Biol. 31:255-265.

Dang, F., Wang, Y., She, J., Lei, Y., Liu, Z., Eulgem, T., Lai, Y., Lin, J., Yu, L., Lei, D., Guan, D., Li, X., Yuan, Q., and He, S. 2014. Overexpression of CaWRKY27, a subgroup IIe WRKY transcription factor of Capsicum annиит, positively regulates tobacco resistance to Ralstonia solanacearum infection. Physiol. Plant. 150:397-411.

Dang, F. F., Wang, Y. N., Yu, L., Eulgem, T., Lai, Y., Liu, Z. Q., Wang, X., Qiu, A. L., Zhang, T. X., Lin, J., Chen, Y. S., Guan, D. Y., Cai, H. Y., Mou, S. L., and He, S. L. 2013. CaWRKY40, a WRKY protein of pepper, plays an important role in the regulation of tolerance to heat stress and resistance to Ralstonia solanacearum infection. Plant Cell Environ. 36:757-774.

De Vos, M., Van Oosten, V. R., Van Poecke, R. M., Van Pelt, J. A., Pozo, M. J., Mueller, M. J., Buchala, A. J., Métraux, J. P., Van Loon, L. C., Dicke, M., and Pieterse, C. M. 2005. Signal signature and transcriptome changes of Arabidopsis during pathogen and insect attack. Mol. PlantMicrobe Interact 18:923-937.

Dong, T., Chen, G., Tian, S., Xie, Q., Yin, W., Zhang, Y., and Hu, Z. 2014. A non-climacteric fruit gene CaMADS-RIN regulates fruit ripening and ethylene biosynthesis in climacteric fruit. PLoS One 9:e95559.

Fammartino, A., Verdaguer, B., Fournier, J., Tamietti, G., Carbonne, F., Esquerré-Tugayé, M. T., and Cardinale, F. 2010. Coordinated transcriptional regulation of the divinyl ether biosynthetic genes in tobacco by signal molecules related to defense. Plant Physiol. Biochem. 48:225-231.

Flores-Cruz, Z., and Allen, C. 2009. Ralstonia solanacearum encounters an oxidative environment during tomato infection. Mol. Plant-Microbe Interact 22:773-782.

Gao, D., Appiano, M., Huibers, R. P., Chen, X., Loonen, A. E., Visser, R. G., Wolters, A. M., and Bai, Y. 2014. Activation tagging of ATHB13 
in Arabidopsis thaliana confers broad-spectrum disease resistance. Plant Mol. Biol. 86:641-653.

Ge, X. X., Liu, Z., Wu, X. M., Chai, L. J., and Guo, W. W. 2015. Genome-wide identification, classification and analysis of HD-ZIP gene family in citrus, and its potential roles in somatic embryogenesis regulation. Gene 574:61-68.

Gehl, C., Kaufholdt, D., Hamisch, D., Bikker, R., Kudla, J., Mendel, R. R., and Hänsch, R. 2011. Quantitative analysis of dynamic protein-protein interactions in planta by a floated-leaf luciferase complementation imaging (FLuCI) assay using binary Gateway vectors. Plant J. 67: 542-553.

Ghanta, S., Bhattacharyya, D., Sinha, R., Banerjee, A., and Chattopadhyay, S. 2011. Nicotiana tabacum overexpressing $\gamma$-ECS exhibits biotic stress tolerance likely through NPR1-dependent salicylic acid-mediated pathway. Planta 233:895-910.

Glazebrook, J. 2005. Contrasting mechanisms of defense against biotrophic and necrotrophic pathogens. Annu. Rev. Phytopathol. 43:205-227.

Goossens, J., Mertens, J., and Goossens, A. 2017. Role and functioning of bHLH transcription factors in jasmonate signalling. J. Exp. Bot. 68: 1333-1347.

Harris, J. C., Hrmova, M., Lopato, S., and Langridge, P. 2011. Modulation of plant growth by HD-Zip class I and II transcription factors in response to environmental stimuli. New Phytol. 190:823-837.

Harris, J. C., Sornaraj, P., Taylor, M., Bazanova, N., Baumann, U., Lovell, B., Langridge, P., Lopato, S., and Hrmova, M. 2016. Molecular interactions of the $\gamma$-clade homeodomain-leucine zipper class I transcription factors during the wheat response to water deficit. Plant Mol. Biol. 90: 435-452.

Hein, I., Gilroy, E. M., Armstrong, M. R., and Birch, P. R. 2009. The zigzag-zig in oomycete-plant interactions. Mol. Plant Pathol. 10:547-562.

Henriksson, E., Olsson, A. S., Johannesson, H., Johansson, H., Hanson, J., Engström, P., and Söderman, E. 2005. Homeodomain leucine zipper class I genes in Arabidopsis. Expression patterns and phylogenetic relationships. Plant Physiol. 139:509-518.

Hong, J. K., Lee, S. C., and Hwang, B. K. 2005. Activation of pepper basic PR-1 gene promoter during defense signaling to pathogen, abiotic and environmental stresses. Gene 356:169-180.

Hu, R., Chi, X., Chai, G., Kong, Y., He, G., Wang, X., Shi, D., Zhang, D., and Zhou, G. 2012. Genome-wide identification, evolutionary expansion, and expression profile of homeodomain-leucine zipper gene family in poplar (Populus trichocarpa). PLoS One 7:e31149.

Hu, T., Ye, J., Tao, P., Li, H., Zhang, J., Zhang, Y., and Ye, Z. 2016. The tomato HD-Zip I transcription factor SlHZ24 modulates ascorbate accumulation through positive regulation of the D-mannose/L-galactose pathway. Plant J. 85:16-29.

Jamir, Y., Guo, M., Oh, H. S., Petnicki-Ocwieja, T., Chen, S., Tang, X., Dickman, M. B., Collmer, A., and Alfano, J. R. 2004. Identification of Pseudomonas syringae type III effectors that can suppress programmed cell death in plants and yeast. Plant J. 37:554-565.

Johannesson, H., Wang, Y., and Engström, P. 2001. DNA-binding and dimerization preferences of Arabidopsis homeodomain-leucine zipper transcription factors in vitro. Plant Mol. Biol. 45:63-73.

Jones, J. D., and Dangl, J. L. 2006. The plant immune system. Nature 444: 323-329.

Jung, H. W., and Hwang, B. K. 2000. Pepper gene encoding a basic $\beta-1,3-$ glucanase is differentially expressed in pepper tissues upon pathogen infection and ethephon or methyl jasmonate treatment. Plant Sci. 159: 97-106.

Jung, H. W., and Hwang, B. K. 2007. The leucine-rich repeat (LRR) protein, CaLRR1, interacts with the hypersensitive induced reaction (HIR) protein, CaHIR1, and suppresses cell death induced by the CaHIR1 protein. Mol. Plant Pathol. 8:503-514.

Jung, H. W., Lim, C. W., Lee, S. C., Choi, H. W., Hwang, C. H., and Hwang, B. K. 2008. Distinct roles of the pepper hypersensitive induced reaction protein gene CaHIRl in disease and osmotic stress, as determined by comparative transcriptome and proteome analyses. Planta 227:409-425.

Kim, D. S., Choi, H. W., and Hwang, B. K. 2014. Pepper mildew resistance locus $\mathrm{O}$ interacts with pepper calmodulin and suppresses Xanthomonas AvrBsT-triggered cell death and defense responses. Planta 240:827-839.

Kovalchuk, N., Chew, W., Sornaraj, P., Borisjuk, N., Yang, N., Singh, R., Bazanova, N., Shavrukov, Y., Guendel, A., Munz, E., Borisjuk, L. Langridge, P., Hrmova, M., and Lopato, S. 2016. The homeodomain transcription factor TaHDZipI-2 from wheat regulates frost tolerance, flowering time and spike development in transgenic barley. New Phytol. 211:671-687.

Kunkel, B. N., and Brooks, D. M. 2002. Cross talk between signaling pathways in pathogen defense. Curr. Opin. Plant Biol. 5:325-331.

Lasserre, E., Godard, F., Bouquin, T., Hernandez, J. A., Pech, J. C., Roby, D., and Balagué, C. 1997. Differential activation of two ACC oxidase gene promoters from melon during plant development and in response to pathogen attack. Mol. Gen. Genet. 256:211-222.

Li, J. B., Luan, Y. S., and Liu, Z. 2015. Overexpression of SpWRKY1 promotes resistance to Phytophthora nicotianae and tolerance to salt and drought stress in transgenic tobacco. Physiol. Plant. 155:248-266.

Liu, Z. Q., Liu, Y. Y., Shi, L. P., Yang, S., Shen, L., Yu, H. X., Wang, R. Z., Wen, J. Y., Tang, Q., Hussain, A., Khan, M. I., Hu, J., Liu, C. L., Zhang, Y. W., Cheng, W., and He, S. L. 2016. SGT1 is required in PcINF1/SRC2-1 induced pepper defense response by interacting with SRC2-1. Sci. Rep. 6: 21651.

Livak, K. J., and Schmittgen, T. D. 2001. Analysis of relative gene expression data using real-time quantitative PCR and the $2^{-\Delta \Lambda \mathrm{C}(\mathrm{T})}$ method. Methods 25:402-408.

Maimbo, M., Ohnishi, K., Hikichi, Y., Yoshioka, H., and Kiba, A. 2007. Induction of a small heat shock protein and its functional roles in Nicotiana plants in the defense response against Ralstonia solanacearum. Plant Physiol. 145:1588-1599.

Manavella, P. A., Dezar, C. A., Bonaventure, G., Baldwin, I. T., and Chan, R. L. 2008. HAHB4, a sunflower HD-Zip protein, integrates signals from the jasmonic acid and ethylene pathways during wounding and biotic stress responses. Plant J. 56:376-388.

Mao, H., Yu, L., Li, Z., Liu, H., and Han, R. 2016. Molecular evolution and gene expression differences within the HD-Zip transcription factor family of Zea mays L. Genetica 144:243-257.

Matsuoka, M., Yamamoto, N., Kano-Murakami, Y., Tanaka, Y., Ozeki, Y., Hirano, H., Kagawa, H., Oshima, M., and Ohashi, Y. 1987. Classification and structural comparison of full-length cDNAs for pathogenesis-related proteins. Plant Physiol. 85:942-946.

Mayda, E., Tornero, P., Conejero, V., and Vera, P. 1999. A tomato homeobox gene (HD-zip) is involved in limiting the spread of programmed cell death. Plant J. 20:591-600.

Meijer, A. H., de Kam, R. J., d'Erfurth, I., Shen, W., and Hoge, J. H. 2000. HD-Zip proteins of families I and II from rice: Interactions and functional properties. Mol. Gen. Genet. 263:12-21.

Merelo, P., Ram, H., Pia Caggiano, M., Ohno, C., Ott, F., Straub, D., Graeff, M., Cho, S. K., Yang, S. W., Wenkel, S., and Heisler, M. G. 2016. Regulation of MIR165/166 by class II and class III homeodomain leucine zipper proteins establishes leaf polarity. Proc. Natl. Acad. Sci. U.S.A. 113:11973-11978.

Müller, C. J., Valdés, A. E., Wang, G., Ramachandran, P., Beste, L., Uddenberg, D., and Carlsbecker, A. 2016. PHABULOSA mediates an auxin signaling loop to regulate vascular patterning in Arabidopsis. Plant Physiol. 170:956-970.

Mur, L. A., Kenton, P., Atzorn, R., Miersch, O., and Wasternack, C. 2006. The outcomes of concentration-specific interactions between salicylate and jasmonate signaling include synergy, antagonism, and oxidative stress leading to cell death. Plant Physiol. 140:249-262.

Naoumkina, M. A., He, X., and Dixon, R. A. 2008. Elicitor-induced transcription factors for metabolic reprogramming of secondary metabolism in Medicago truncatula. BMC Plant Biol. 8:132.

Oh, S. K., Park, J. M., Joung, Y. H., Lee, S., Chung, E., Kim, S. Y., Yu, S. H., and Choi, D. 2005. A plant EPF-type zinc-finger protein, CaPIF1, involved in defence against pathogens. Mol. Plant Pathol. 6:269-285.

Oh, S. K., Yoon, J., Choi, G. J., Jang, H. A., Kwon, S. Y., and Choi, D. 2013. Capsicum annuum homeobox 1 (CaHB1) is a nuclear factor that has roles in plant development, salt tolerance, and pathogen defense. Biochem. Biophys. Res. Commun. 442:116-121.

Palena, C. M., Gonzalez, D. H., and Chan, R. L. 1999. A monomer-dimer equilibrium modulates the interaction of the sunflower homeodomain leucine-zipper protein Hahb-4 with DNA. Biochem. J. 341:81-87.

Pétriacq, P., Stassen, J. H., and Ton, J. 2016. Spore density determines infection strategy by the plant pathogenic fungus Plectosphaerella cucumerina. Plant Physiol. 170:2325-2339.

Pieterse, C. M., Van der Does, D., Zamioudis, C., Leon-Reyes, A., and Van Wees, S. C. 2012. Hormonal modulation of plant immunity. Annu. Rev. Cell Dev. Biol. 28:489-521.

Qiu, A., Liu, Z., Li, J., Chen, Y., Guan, D., and He, S. 2016. The ectopic expression of CaRopl modulates the response of tobacco plants to Ralstonia solanacearum and aphids. Front. Plant Sci. 7:1177.

Romani, F., Ribone, P. A., Capella, M., Miguel, V. N., and Chan, R. L. 2016 A matter of quantity: Common features in the drought response of transgenic plants overexpressing HD-Zip I transcription factors. Plant Sci. 251:139-154.

Roodbarkelari, F., and Groot, E. P. 2017. Regulatory function of homeodomain-leucine zipper (HD-ZIP) family proteins during embryogenesis. New Phytol. 213:95-104.

Sarowar, S., Kim, Y. J., Kim, E. N., Kim, K. D., Hwang, B. K., Islam, R., and Shin, J. S. 2005. Overexpression of a pepper basic pathogenesis- 
related protein 1 gene in tobacco plants enhances resistance to heavy metal and pathogen stresses. Plant Cell Rep. 24:216-224.

Schena, M., and Davis, R. W. 1994. Structure of homeobox-leucine zipper genes suggests a model for the evolution of gene families. Proc. Natl. Acad. Sci. U.S.A. 91:8393-8397.

Shen, L., Liu, Z., Yang, S., Yang, T., Liang, J., Wen, J., Liu, Y., Li, J., Shi, L., Tang, Q., Shi, W., Hu, J., Liu, C., Zhang, Y., Lin, W., Wang, R., Yu, H., Mou, S., Hussain, A., Cheng, W., Cai, H., He, L., Guan, D., Wu, Y. and He, S. 2016b. Pepper CabZIP63 acts as a positive regulator during Ralstonia solanacearum or high temperature-high humidity challenge in a positive feedback loop with CaWRKY40. J. Exp. Bot. 67:2439-2451.

Shen, L., Yang, S., Yang, T., Liang, J., Cheng, W., Wen, J., Liu, Y., Li, J., Shi, L., Tang, Q., Shi, W., Hu, J., Liu, C., Zhang, Y., Mou, S., Liu, Z., Cai, H., He, L., Guan, D., Wu, Y., and He, S. 2016a. CaCDPK15 positively regulates pepper responses to Ralstonia solanacearum inoculation and forms a positive-feedback loop with CaWRKY40 to amplify defense signaling. Sci. Rep. 6:22439.

Sohn, S. I., Kim, Y. H., Kim, B. R., Lee, S. Y., Lim, C. K., Hur, J. H., and Lee, J. Y. 2007. Transgenic tobacco expressing the $h r p N(E P)$ gene from Erwinia pyrifoliae triggers defense responses against botrytis cinerea. Mol. Cells 24:232-239.

Spoel, S. H., Johnson, J. S., and Dong, X. 2007. Regulation of tradeoffs between plant defenses against pathogens with different lifestyles. Proc. Natl. Acad. Sci. U.S.A. 104:18842-18847.

Takahashi, Y., Uehara, Y., Berberich, T., Ito, A., Saitoh, H., Miyazaki, A., Terauchi, R., and Kusano, T. 2004. A subset of hypersensitive response marker genes, including $H S R 203 J$, is the downstream target of a spermine signal transduction pathway in tobacco. Plant J. 40:586-595.

Tsuda, K., and Katagiri, F. 2010. Comparing signaling mechanisms engaged in pattern-triggered and effector-triggered immunity. Curr. Opin. Plant Biol. 13:459-465.

Tsuda, K., Sato, M., Stoddard, T., Glazebrook, J., and Katagiri, F. 2009. Network properties of robust immunity in plants. PLoS Genet. 5:e1000772.

Wang, Y., Dang, F., Liu, Z., Wang, X., Eulgem, T., Lai, Y., Yu, L., She, J., Shi, Y., Lin, J., Chen, C., Guan, D., Qiu, A., and He, S. 2013. CaWRKY58, encoding a group I WRKY transcription factor of Capsicum annuum, negatively regulates resistance to Ralstonia solanacearum infection. Mol. Plant Pathol. 14:131-144.

Wang, Y., Wang, H., Ma, Y., Du, H., Yang, Q., and Yu, D. 2015. Identification of transcriptional regulatory nodes in soybean defense networks using transient co-transactivation assays. Front. Plant Sci. 6:915.
Ward, E. R., Payne, G. B., Moyer, M. B., Williams, S. C., Dincher, S. S. Sharkey, K. C., Beck, J. J., Taylor, H. T., Ahl-Goy, P., Meins, F., and Ryals, J. A. 1991. Differential regulation of $\beta$-1,3-glucanase messenger RNAs in response to pathogen infection. Plant Physiol. 96:390-397.

Xu, Z. S., Chen, M., Li, L. C., and Ma, Y. Z. 2011. Functions and application of the AP2/ERF transcription factor family in crop improvement. J. Integr. Plant Biol. 53:570-585.

Yan, T., Chen, M., Shen, Q., Li, L., Fu, X., Pan, Q., Tang, Y., Shi, P. Lv, Z., Jiang, W., Ma, Y. N., Hao, X., Sun, X., and Tang, K. 2017. HOMEODOMAIN PROTEIN 1 is required for jasmonate-mediated glandular trichome initiation in Artemisia annua. New Phytol. 213: 1145-1155.

Yi, H. S., Yang, J. W., and Ryu, C. M. 2013. ISR meets SAR outside: Additive action of the endophyte Bacillus pumilus INR7 and the chemical inducer, benzothiadiazole, on induced resistance against bacterial spot in field-grown pepper. Front Plant Sci 4:122.

Zhang, C. H., Ma, R. J., Shen, Z. J., Sun, X., Korir, N. K., and Yu, M. L. 2014a. Genome-wide analysis of the homeodomain-leucine zipper (HD-ZIP) gene family in peach (Prunus persica). Genet. Mol. Res. 13: 2654-2668.

Zhang, J. Y., Qiao, Y. S., Lv, D., Gao, Z. H., Qu, S. C., and Zhang, Z. 2012a. Malus hupehensis NPRl induces pathogenesis-related protein gene expression in transgenic tobacco. Plant Biol Stuttg 14 (Suppl 1):46-56.

Zhang, L., Li, Y., Lu, W., Meng, F., Wu, C. A., and Guo, X. 2012b. Cotton GhMKK5 affects disease resistance, induces HR-like cell death, and reduces the tolerance to salt and drought stress in transgenic Nicotiana benthamiana. J. Exp. Bot. 63:3935-3951.

Zhang, Z., Chen, X., Guan, X., Liu, Y., Chen, H., Wang, T., Mouekouba, L. D., Li, J., and Wang, A. 2014b. A genome-wide survey of homeodomainleucine zipper genes and analysis of cold-responsive HD-Zip I members' expression in tomato. Biosci. Biotechnol. Biochem. 78:1337-1349.

Zhao, Y., Ma, Q., Jin, X., Peng, X., Liu, J., Deng, L., Yan, H., Sheng, L., Jiang, H., and Cheng, B. 2014. A novel maize homeodomain-leucine zipper (HD-Zip) I gene, Zmhdz10, positively regulates drought and salt tolerance in both rice and Arabidopsis. Plant Cell Physiol. 55:1142-1156.

\section{AUTHOR-RECOMMENDED INTERNET RESOURCE}

cNLS Mapper open source software: http://nls-mapper.iab.keio.ac.jp/cgi-bin/NLS_Mapper_form.cgi 